\title{
Structural Analysis for the American Airlines Flight 587 Accident Investigation - Global Analysis
}

\author{
Richard D. Young* \\ NASA Langley Research Center, Hampton, VA 23681-2199 \\ Andrew E. Lovejoy ${ }^{\dagger}$ \\ Analytical Services \& Materials, Inc., Hampton, VA 23666-1340 \\ Mark. W. Hilburger ${ }^{\ddagger}$ and David F. Moore ${ }^{\S}$ \\ NASA Langley Research Center, Hampton, VA 23681-2199
}

\begin{abstract}
NASA Langley Research Center (LaRC) supported the National Transportation Safety Board (NTSB) in the American Airlines Flight 587 accident investigation due to LaRC's expertise in high-fidelity structural analysis and testing of composite structures and materials. A Global Analysis Team from LaRC reviewed the manufacturer's design and certification procedures, developed finite element models and conducted structural analyses, and participated jointly with the NTSB and Airbus in subcomponent tests conducted at Airbus in Hamburg, Germany. The Global Analysis Team identified no significant or obvious deficiencies in the Airbus certification and design methods. Analysis results from the LaRC team indicated that the most-likely failure scenario was failure initiation at the right rear main attachment fitting (lug), followed by an unstable progression of failure of all fin-to-fuselage attachments and separation of the VTP from the aircraft. Additionally, analysis results indicated that failure initiates at the final observed maximum fin loading condition in the accident, when the VTP was subjected to loads that were at minimum 1.92 times the design limit load condition for certification. For certification, the VTP is only required to support loads of 1.5 times design limit load without catastrophic failure. The maximum loading during the accident was shown to significantly exceed the certification requirement. Thus, the structure appeared to perform in a manner consistent with its design and certification, and failure is attributed to VTP loads greater than expected.
\end{abstract}

\section{Introduction}

$\mathrm{O}$ November 12, 2001, an Airbus 300-600R being operated as American Airlines Flight 587 crashed soon after take-off from John F. Kennedy airport in New York City, killing all 260 persons aboard and 5 on the ground. The plane's composite vertical stabilizer and rudder (referred to herein as the Vertical Tail Plane or VTP) separated from the aircraft before it impacted the ground. Initial analyses indicated that this accident was the first commercial aircraft crash that involved the failure of a primary structure made from composite materials. NASA Langley Research Center (LaRC) was requested by the National Transportation Safety Board (NTSB) to support the accident investigation because of LaRC's expertise in high-fidelity structural analysis and testing of composite structures and materials. In coordination with the NTSB and under the technical guidance of Dr. James H. Starnes, Jr. of NASA LaRC, technical expertise was provided for several aspects of the investigation that included global analysis of the composite vertical tail fin and rudder. This paper presents a summary of the NASA AA587 Global Analysis Team results for the American Airlines Flight 587 accident investigation. ${ }^{1}$

The charter established by the NTSB for the NASA Global Analysis Team was as follows:

The team shall address the following objectives:

- Review of Airbus certification process: testing, analysis and design procedures

\footnotetext{
* Senior Research Engineer, Mechanics of Structures and Materials Branch, Senior Member AIAA

${ }^{\dagger}$ Research Scientist, Senior Member AIAA, Member ASME

${ }^{*}$ Aerospace Engineer, Mechanics of Structures and Materials Branch, Member AIAA

${ }^{\S}$ Aerospace Engineer, Structural \& Thermal Analysis Branch, Member ASME
} 
- Develop and interrogate failure scenarios

- Provide loads to Local Analysis Team to perform strength analyses

- Conduct failure sequence analyses for most likely failure scenario (and correlate predicted damage with the physical evidence)

- Provide evidence to assess whether the structure performed as intended

To address this charter, the NASA Global Analysis Team reviewed the manufacturer's design and certification procedures, developed finite element models and conducted structural analyses, and participated jointly with the NTSB and Airbus in subcomponent tests conducted at Airbus in Hamburg, Germany. Throughout the investigation, the NASA team worked closely with the investigation teams at the NTSB and Airbus. Several of the investigation analysis efforts were developed independently by the NASA and Airbus teams and had common objectives. However, in some cases, different analysis approaches were used and served to verify the predicted results. The NASA team received initial finite element models from Airbus. These initial models were modified by the NASA team, as deemed necessary, and then verified to the point that the NASA team felt confident in the validity of the models. For the analysis efforts that were common between the NASA and Airbus teams, the NASA team's efforts may be used to independently verify Airbus results. In addition to conducting standard linear analyses for the most likely failure scenario, the NASA team also considered variations in analysis procedures and model details to determine if alternative failure scenarios would be indicated. The following text outlines the sequence of activities conducted by the NASA Global Analysis Team, and the findings of the team are reported in the body of this paper.

The NASA team conducted a review of Airbus design and certification documents and the results of this review are described in Section II. The team also conducted a review of the Airbus fin and rudder NASTRAN ${ }^{2}$ models that were delivered to NASA, and made a number of model modifications that were implemented in an attempt to improve model fidelity, enable geometrically nonlinear analysis, and enable dynamic analysis (see Section III). Failure scenarios were developed based upon the physical evidence ${ }^{3,4}$ and an initial assessment of the predicted critical reserve factors of the VTP subjected to accident load conditions (Section IV). The most likely failure scenario was identified and validated by conducting a subcomponent test with loading based on analysis results of the VTP at the final observed maximum fin loading condition, as described in Section V. The analysis included a global/local iterative procedure to effectively embed a refined, local three-dimensional finite element model into the global model. ${ }^{5}$ In Section VI, the VTP hardware failure sequences associated with the most likely failure scenario that are predicted using both static and dynamic analyses are described, and these results are compared to the physical evidence.

\section{Review of Airbus A300-600 Certification}

One of the first tasks of the NASA Global Analysis Team was to review the Airbus certification and design methods used in the design of the composite VTP of the A300-600 Airplane. Specific goals of the review included a review of the Airbus models and analysis methods, reserve factor calculations, and supporting test data used to develop the design allowables. Airbus design justification documents were reviewed to obtain familiarity with the design process and reserve factor calculations, and selected reserve factors were recalculated to verify the Airbus results. This review was conducted to look for errors in modeling, analysis, or testing that would have resulted in an unconservative design.

The NASA Global Analysis Team possesses expertise in high-fidelity analysis and testing of composite structures. The team utilized this expertise to review the Airbus design and certification documents. The certification review included examination of the procedures used for establishing design allowables through coupon and subcomponent tests that were validated using full-scale structural tests to failure (see Fig. 1, that shows typical document content). The review also fostered an understanding of the Airbus allowable calculations, e.g., material failure criteria and reserve factor calculations, in order for the team to calculate meaningful reserve factors and failure predictions for the accident loading conditions. Specifically, material failure criteria and reserve factors were used during the investigation to determine regions that may exhibit failure. It should be noted that these failure allowbles and reserve factors are, in general, very conservative, are used during the design process to indicate satisfaction of design requirements, and do not necessarily indicate the presence of failure. Furthermore, these allowables and factors were used in the investigation to identify the most likely locations of failure.

NASA's examination of the Airbus design allowable and reserve factor processes determined that the Airbus analysis, design and testing procedures were complete and comprehensive, utilizing a well-defined building block approach. Because no significant or obvious deficiencies were identified, the NASA team felt confident using the design allowables, failure calculations and reserve factor calculations to conduct the accident investigation. 


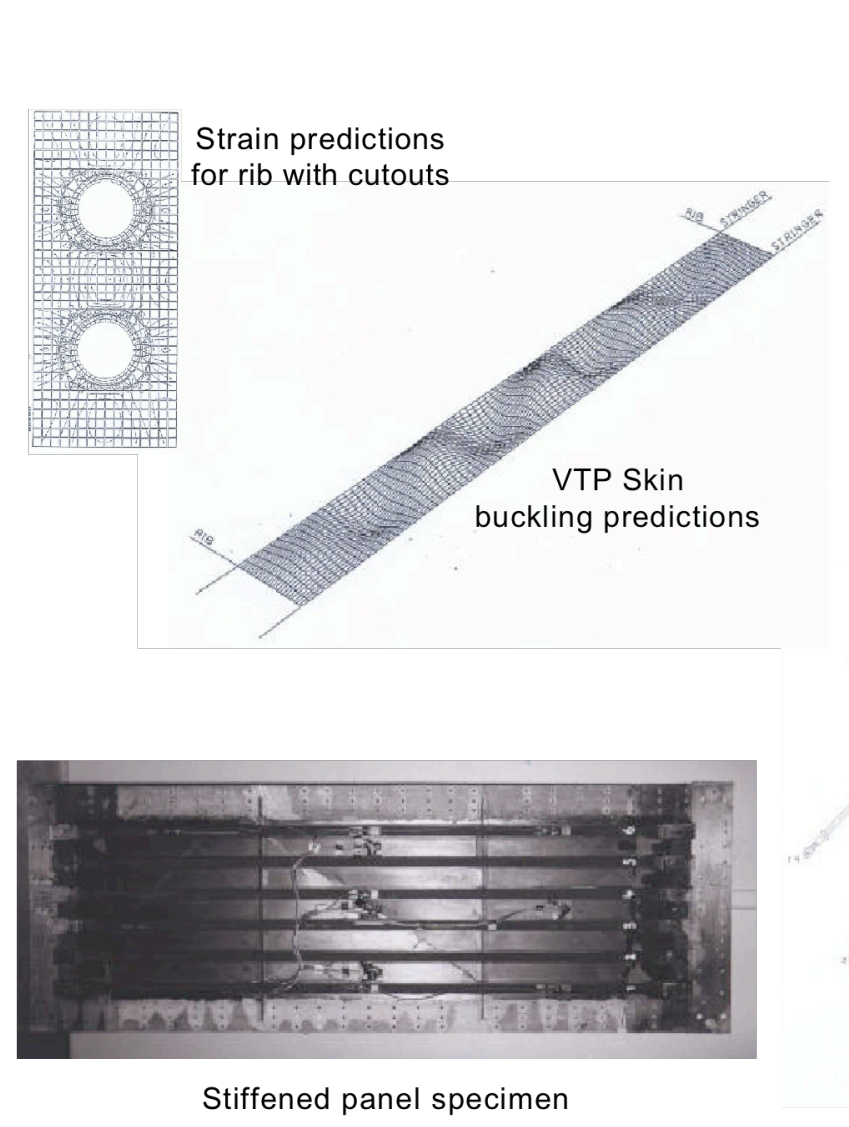

a) Coupon Tests, Subcomponent panel tests, Local detail models

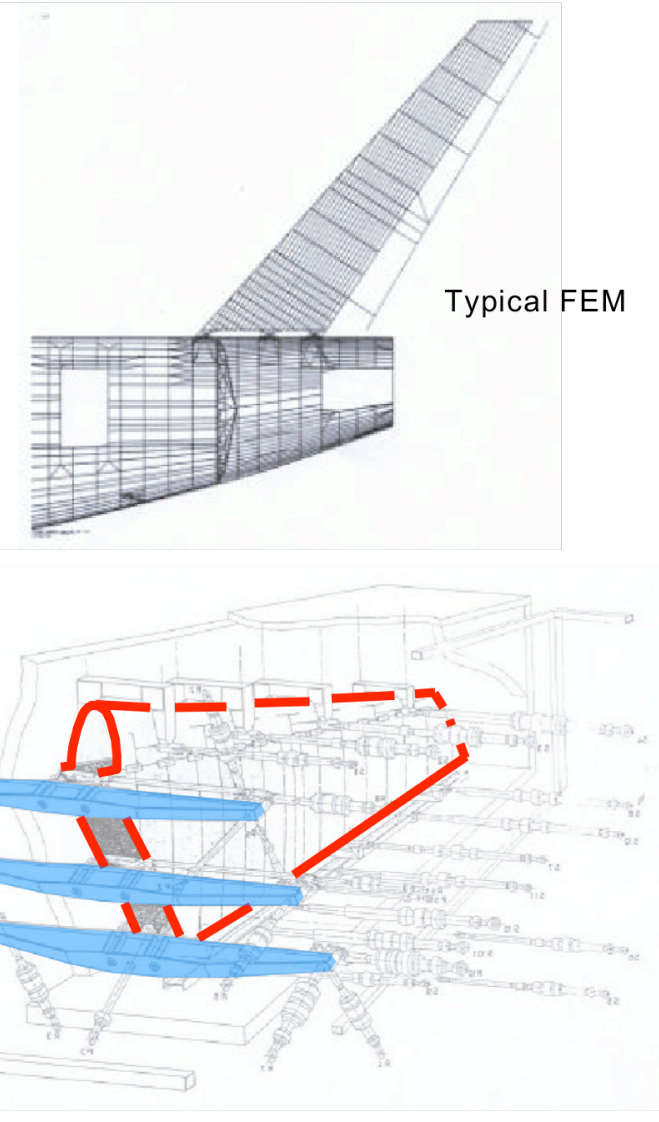

Full-scale test configuration

b) Airbus global FEM, Full-scale component tests

Figure 1: Airbus building-block approach to design allowable development.

However, during the review process, two items of concern with the certification process were identified that were subsequently addressed within the investigation. These certification concerns were as follows:

1) The validity of the full-scale fin certification test was questioned as to whether the loading applied in the fullscale fin test was representative of the actual aircraft loading. The full-scale fin test was conducted with the fin off the aircraft and the distribution of applied loads was prescribed exactly from the analysis. Thus, the validity of the test loadingwas dependent on the validity of the global finite-element model. In addition, the load-introduction structure may have introduced bending moments at the main-attachment fittings, or lugs, that are different than what the fuselage frames would have introduced.

2) The lug-strength allowables applied during design and certification were resultant forces, and did not take into consideration directly the effect of bending moments on the strength of the fin-to-fuselage attachments, and certification analyses were not detailed enough to quantify the bending moments at the lugs. Detailed strength analysis conducted by the NASA AA587 Local Analysis Team has shown that the bending moment at the rear lug attachment influences the failure strength of the lug.

The first concern was addressed during the model verification activities (see Section III) by conducting sensitivity analyses to validate the test load conditions. The second concern was addressed by examining the global attachment pin moment and rotation results and the local progressive failure analysis results. ${ }^{5}$ The team determined that pin moments are not an independent parameter, and therefore, the Airbus method for establishing the strength allowables inherently included moment effects. 


\section{Model Development and Validation}

\section{A. Model Modification and Convergence}

The NASA team conducted detailed model refinement and model validation activities. Airbus provided NASA with a finite element model for the tail structure in March of 2002, which is shown in Fig. 2. This model included changes incorporated by Airbus after the AA587 accident, such as mesh refinement, particularly in the rudder. The Airbus tail structure model comprises three major components; the fuselage section, the vertical stabilizer (fin), and the rudder. Together, the fin and rudder make up the VTP that is connected to the fuselage via 12 attachment fittings (6 lugs and 6 shear yokes). Descriptions of these components are provided in Ref. 1. The finite element model was reviewed to gain familiarity with the model, and then the model was compared to design drawings supplied by Airbus. Comparison was limited to regions of interest, such as areas of observed failure in the accident

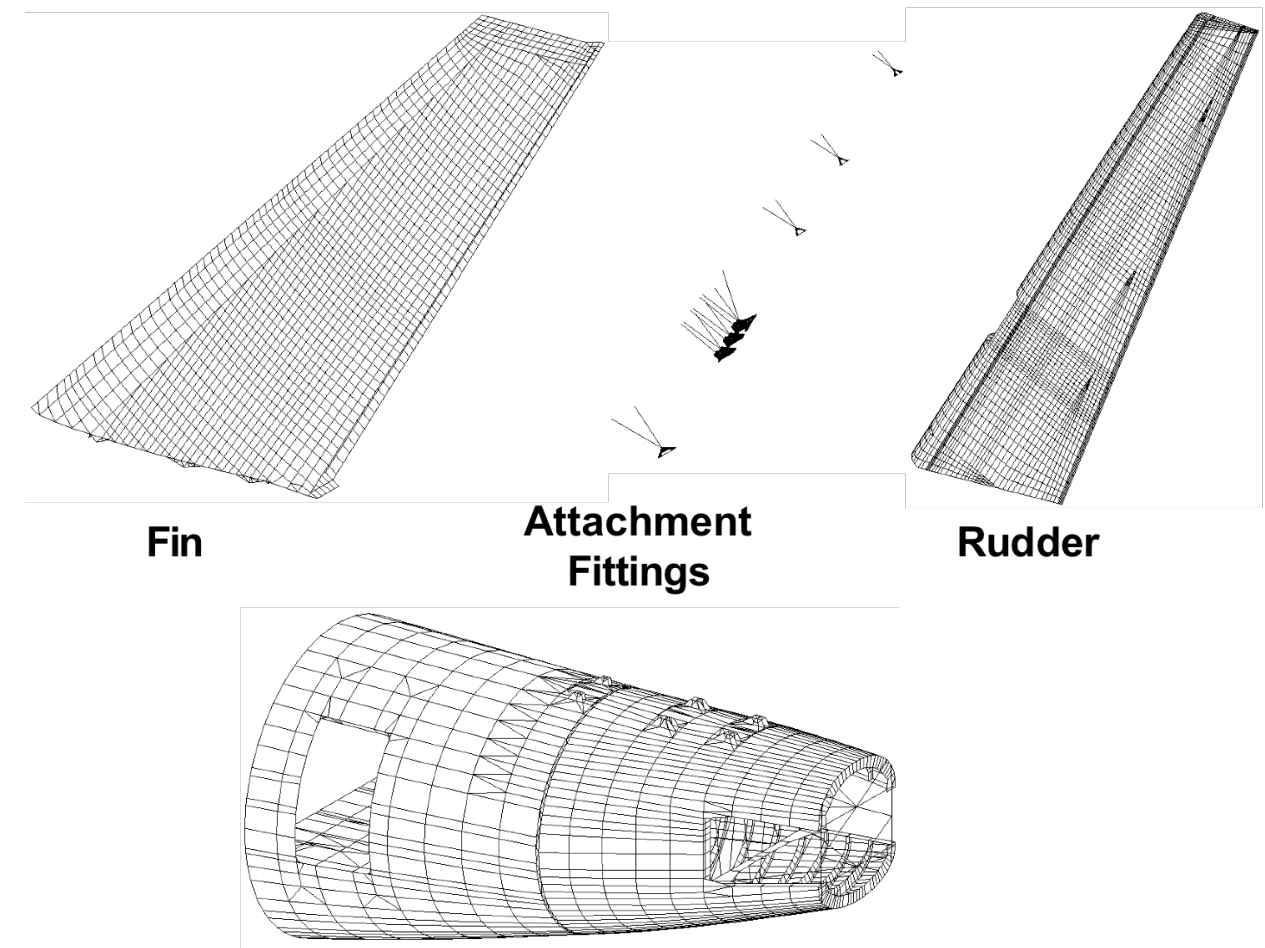

\section{Fuselage}

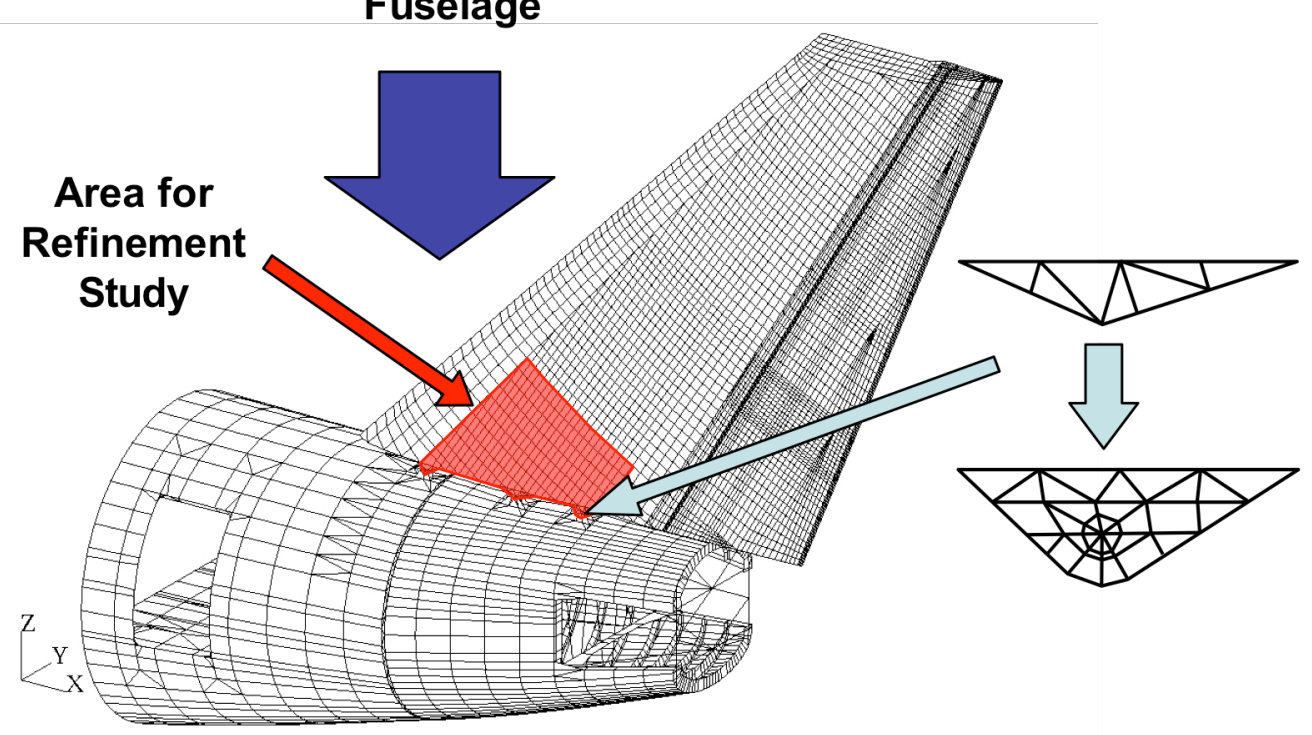

Figure 2: Finite element model of the A300-600 tail section (minus horizontal stabilizer). 
aircraft and in regions where detailed modeling was present, and included the lugs, spar attachment points and the rudder skins. The Airbus model appeared to accurately represent the as-designed structure. An understanding of the Airbus analysis model and method was established by replicating the results for 15 load cases supplied by Airbus, providing a baseline from which model modifications were made to conduct further studies.

A number of model modifications were implemented to improve model accuracy in specific regions and to enable additional analyses, including enabling nonlinear analysis capability, mass adjustment, and lug mesh refinement. The original model supplied by Airbus was not able to support nonlinear analyses in NASTRAN, therefore, a major effort was expended to introduce nonlinear capability into the model. To enable the nonlinear analysis, numerous PATRAN ${ }^{6}$ Command Language (PCL) functions were written to automatically incorporate all necessary changes. The nonlinear capable model mesh remained the same as that for the linear model, however, many elements were changed to elements that are formulated to run in a NASTRAN nonlinear analysis. Next, the mass definitions of the global model were adjusted to permit accurate modal and transient analyses since the model supplied by Airbus was not intended for analyses requiring accurate mass modeling. Modifications were also made to the FEM of the VTP lugs to allow more accurate representation of the thickness distributions and to capture bending response. Additionally, the lug was modified to introduce loads through multiple nodes instead of one. The original Airbus FEM and the final NASA FEM for the right rear lug are shown in Fig. 2. Model modifications were implemented, and then the results from the modified models were interrogated and their convergence and accuracy were assessed.

The response of the lower portion of the fin was examined for convergence through a mesh refinement study in which a large lower portion of the fin was refined. The location of this refinement region is shown in Fig. 2. The region was refined from the baseline model consisting of approximately 21600 nodes and 40000 elements to a refined model consisting of approximately 50600 nodes and 75600 elements. Refinement encompassed all fin components from the lugs up to Rib 5, and was bounded by the front and rear spars, which were included in the refinement. The results of the refinement study were used to demonstrate convergence of the predicted attachment loads and skin strains. It was observed that the mesh refinement has very little effect on the VTP response and it was concluded that the coarse model was sufficient to provide converged results for the required accident loading conditions. Details about all model modifications and the convergence study can be found in Ref. 1 .

\section{B. Linear vs. Nonlinear Analysis}

There was a concern that geometrically nonlinear behavior would affect the response of the VTP under the accident loading, particularly at the maximum loading condition. Therefore, using the nonlinear capable model, geometrically linear and nonlinear analysis results were examined to determine if the VTP exhibited geometrically nonlinear behavior when subjected to the maximum accident loading condition. In particular, VTP deformations, fin skin stress resultants, and main attachment forces and moments predicted for linear and nonlinear analyses were compared. The tip deflections were $608.11 \mathrm{~mm}$ and $606.95 \mathrm{~mm}$ for the linear and nonlinear analyses, respectively, a difference of $-0.2 \%$. Similarly, the right rear lug forces predicted with the linear and nonlinear analyses showed a difference of approximately $5 \%$, and the fin skin force resultants for the two analyses were within $3 \%$. For the most part, there were no other appreciable differences found between the results for the linear and nonlinear analyses. Therefore, linear analysis was deemed sufficient to examine the general response of the VTP structure.

\section{Model Validation}

Airbus conducted a finite element model validation study as a part of their design justification process by comparing results from several full-scale VTP tests with their analysis results. The full-scale test results from a test conducted in 1985 were used to validate the fin portion of the FEM used in the investigation. A sketch of the test setup is illustrated in Fig. 3, with the fin edges outlined in red and the lug load introduction beams highlighted in blue. The main objective was to verify that the FEM accurately predicts the over-all global response (e.g., global deformations) and local response characteristics in the acreage of fin skin panel (e.g., local strains to ensure structural stiffnesses and load paths were accurately modeled. Predicted and measured displacements that characterize the front and rear spar bending response indicated good correlation as shown in Fig. 4. In addition, predicted and measured chordwise strain distributions were compared (see Fig. 5), as were bending strains in the fin skin panels determined from back-to-back strain gage pairs (see Fig. 6). The predicted attachment point reaction forces at the test rupture load showed that the left rear lug load $\mathrm{F}_{\mathrm{xz}}$ (the resultant in-plane load in the lug) is 917,070 $\mathrm{N}$, as compared to $902,000 \mathrm{~N}$ for the tension rupture load as measured in the test, an error of less than $2 \%$. In general, all results showed good correlation between the test and analysis, and indicate that the overall character of load distribution and stiffness in the fin was accurately predicted by the finite element model. 


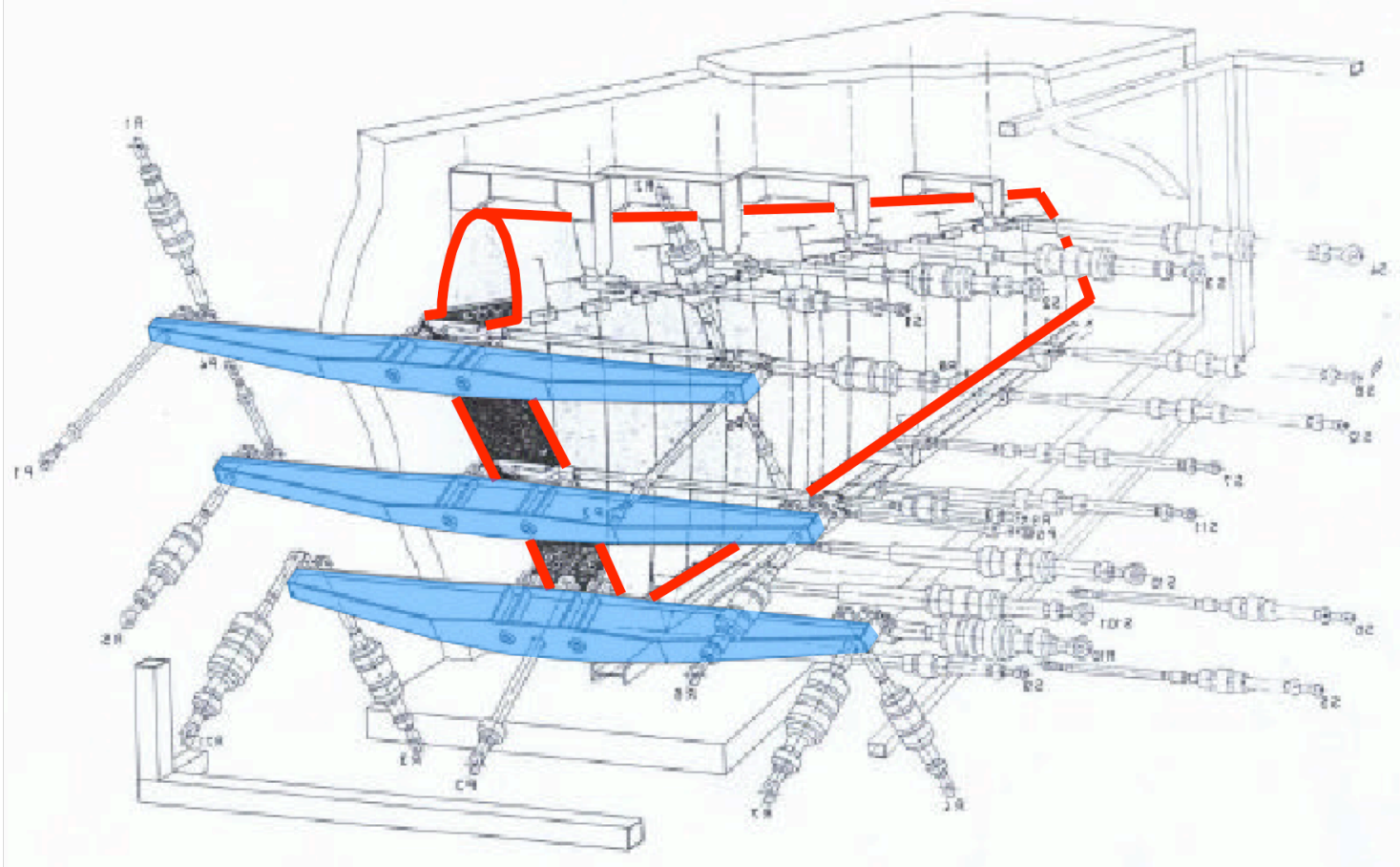

Figure 3: Airbus fin test setup (fin outlined in red, load introduction beams highlighted in blue).

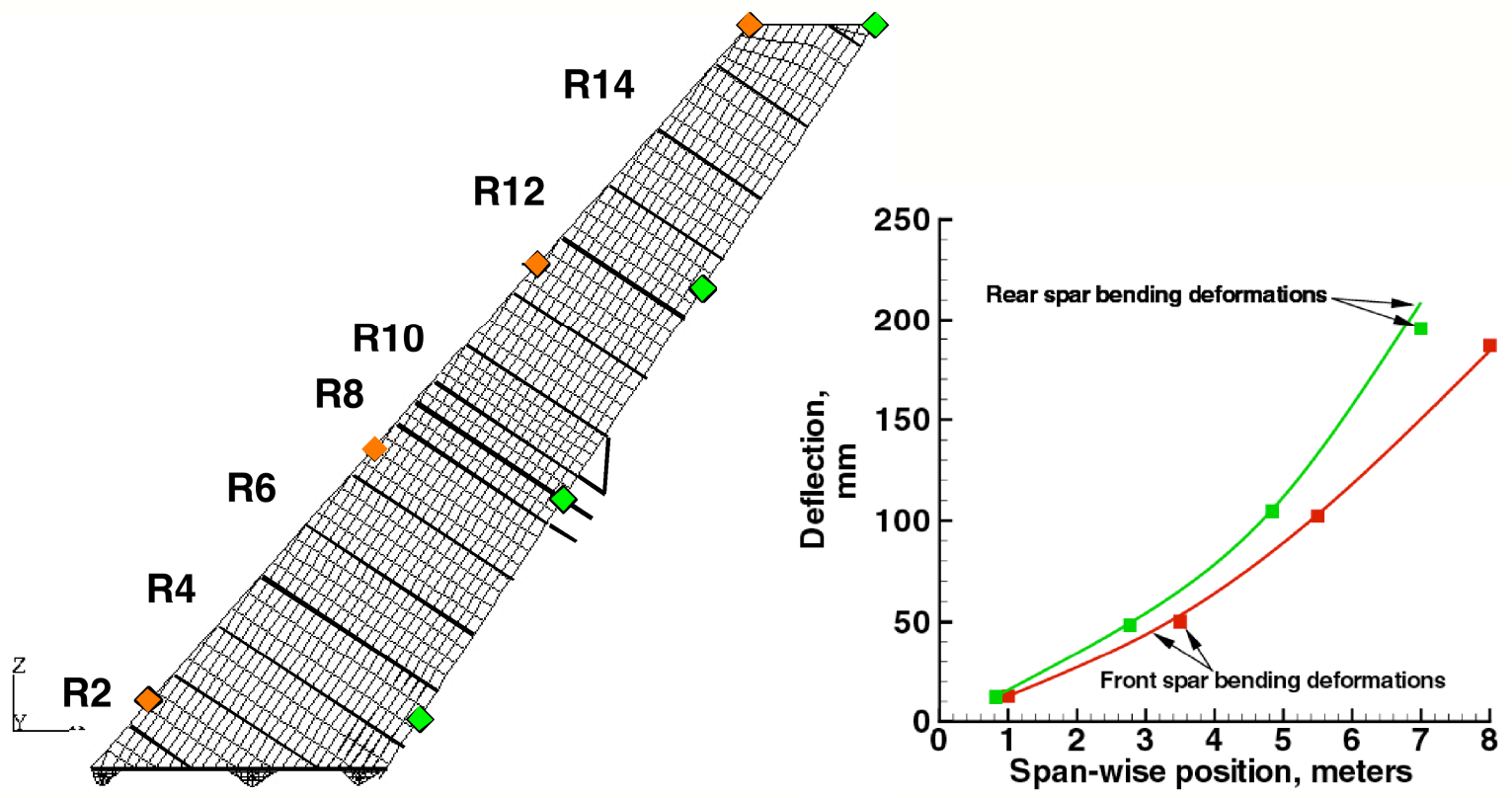

Figure 4: Comparison of full-scale test and analysis fin spar bending lines.

The main attachment loads that were used in the full-scale test were prescribed based on global finite element analysis results. However, the fin/fuselage attachment represents a statically-indeterminate structure. Therefore, the load distribution at the connection points will be dependent upon the geometry and stiffness of the structure. The stiffness of the fin model was demonstrated by comparison of fin responses to the full-scale test, as described above. Sensitivity studies were conducted to examine the dependency of the attachment loads on the fin lug and fuselage stiffnesses. One study focused on the stiffness variations in the lug region of the fin, and the other on stiffness variations in the fuselage. For the regions shown in Fig. 7, the fin stiffness was decreased by $20 \%$ for each region independently, then simultaneously. The fuselage stiffness was studied with the fuselage locally rigidized between the clevis pairs to represent the load introduction beams in the full-scale test (see Fig. 3) and with a fully rigid 


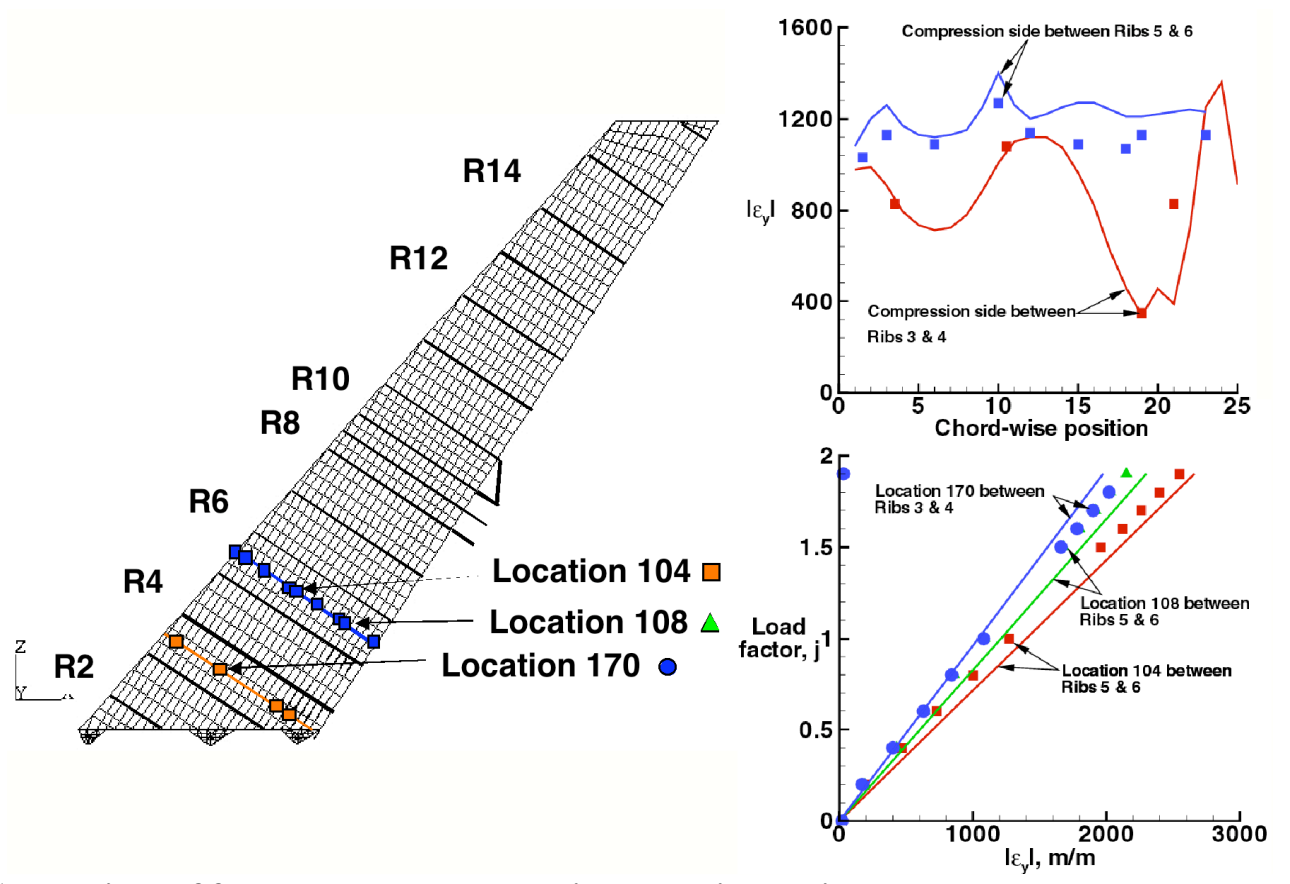

Figure 5: Comparison of full-scale test and analysis chordwise strains.

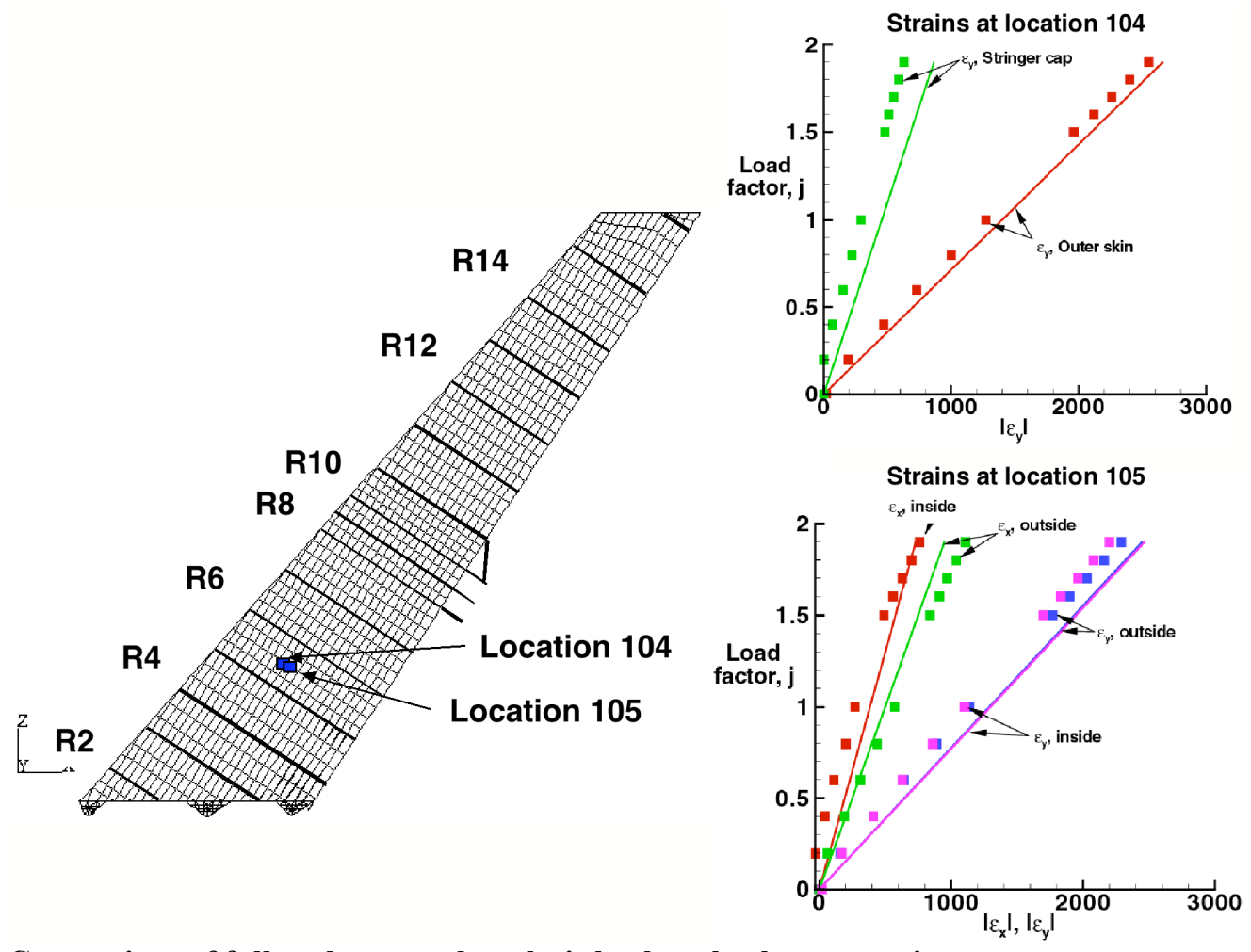

Figure 6: Comparison of full-scale test and analysis back-to-back gage strains.

fuselage. These studies indicated that while there were significant changes in local strains and in tip deflection, as a result of changes in VTP rigid body motion, the attachment loads changed very little. Therefore, it was determined that the load distribution at the attachments is primarily geometry driven so that use of the attachment loads from the global analysis for the off-fuselage full-scale fin test is valid.

Based upon the convergence and validation efforts, it was determined that the finite element model could be used to accurately evaluate the response of the VTP for the accident load conditions to interrogate failure scenarios. 


\section{Failure Scenario Development and Validation}

Five failure scenarios were defined based on the physical evidence and the initial assessment of the critical reserve factors. The critical reserve factors were calculated from the analysis results of the pristine VTP subjected to the accident maximum upset load condition using the modified model described in Section III. Accident flight loads used in the failure scenario investigation were derived from the flight data recorder (FDR) data by Airbus through a procedure verified by the NTSB, and were provided to NASA. The physical evidence of the AA587 VTP has been photographed and documented by the NTSB. Two of these documents were submitted as part of the public docket; Exhibit No. 15-A designated with Docket No. SA-522 and submitted on October 7, $2002,{ }^{3}$ and the Structures Group Chairman's

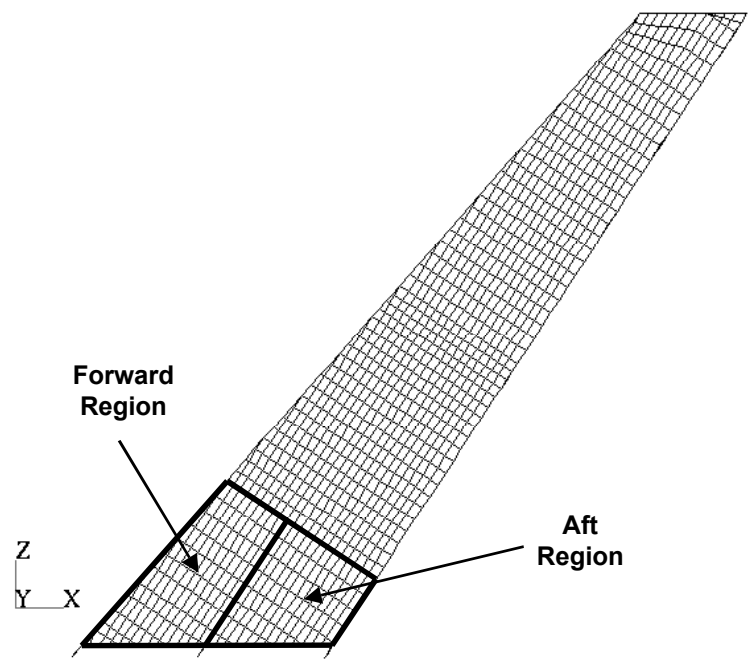

Figure 7: Regions of stiffness reduction for fin stiffness sensitivity study. Factual Report DCA02MA001 that was included in the public docket and revised on December 16, 2002. ${ }^{4}$ Figs. 8 and 9 show pictures of typical fin and rudder damage, and Fig. 10 shows a sketch of the rudder damage. The five

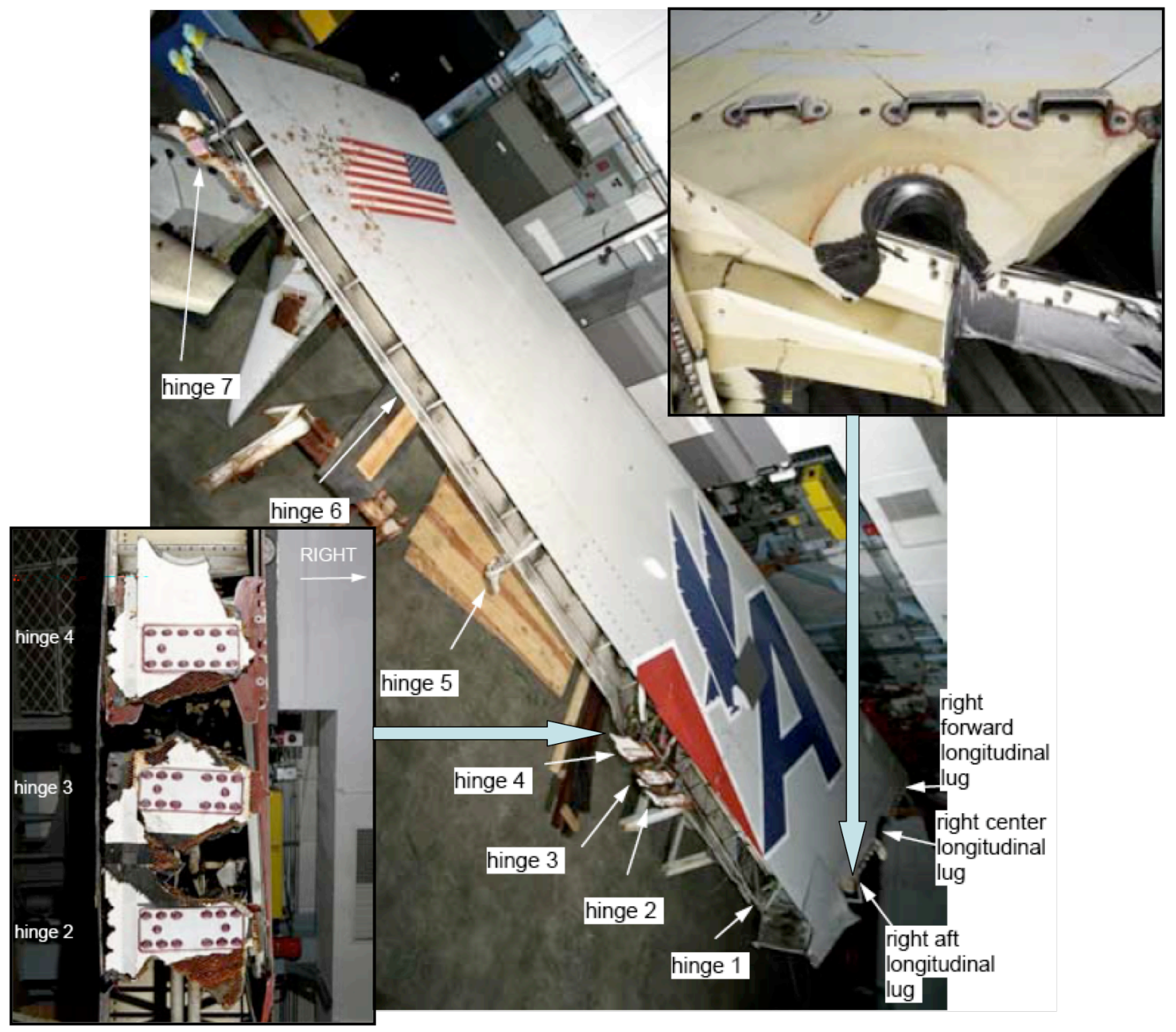

Figure 8: Fin and rudder hinge line damage of AA587. 


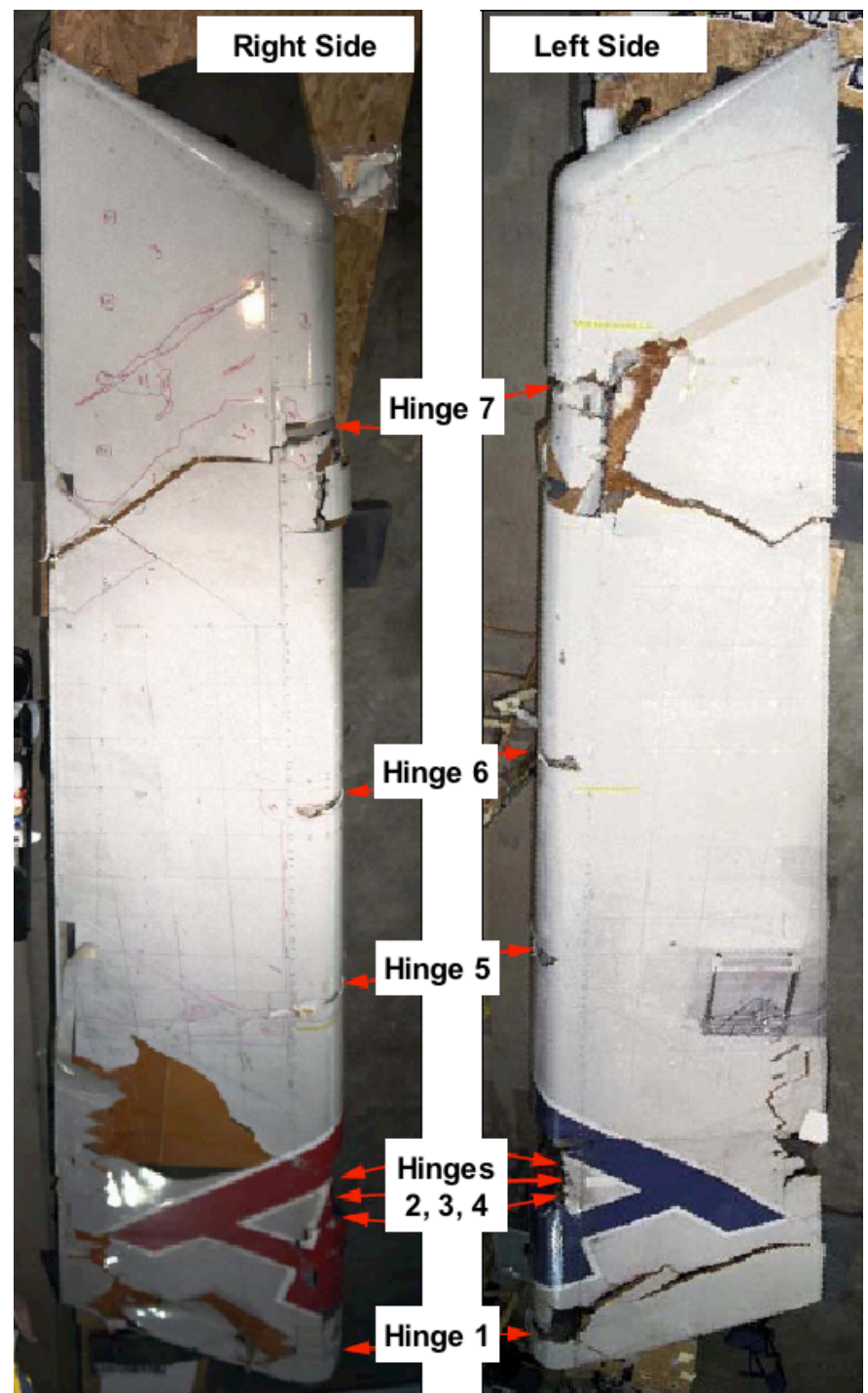

Figure 9: Rudder damage of AA587.

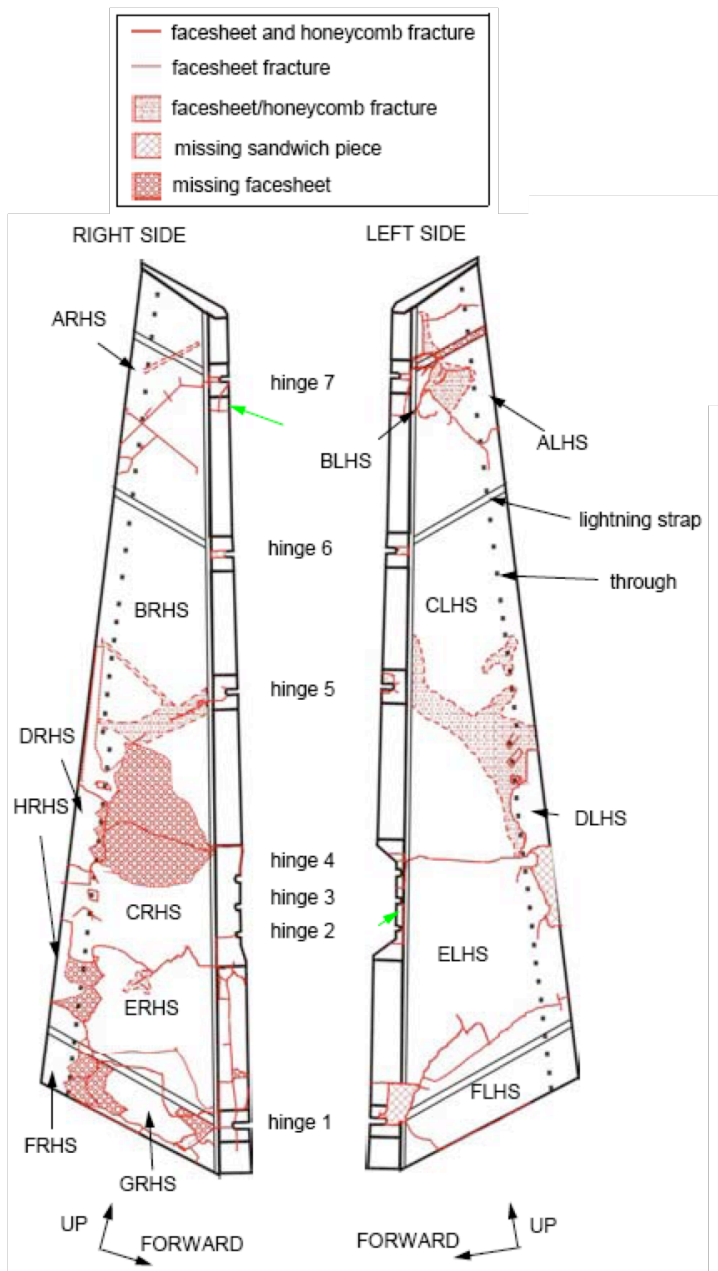

Figure 10: Sketch of rudder damage of AA587.

scenarios that were identified and examined are: 1) main attachment fitting failure, 2) buckling of fin box structure that causes main attachment fitting failure, rudder hinge line failure, or rudder failure,

3) rudder skin failure at the ply-drop detail near the reinforced actuator region, 4) actuation of the bent hinge line that causes rudder fracture or rudder hinge line failure, 5) flutter of the VTP that results from delamination of the rudder skin. The five failure scenarios considered herein were evaluated using various analyses, and the models, analysis methods used and conclusions for these scenarios are discussed in the following subsections.

\section{A. Main Attachment Fitting: Pristine and Pre-existing Damage}

Main attachment fitting failure was examined using the full tail structure model. Flight simulation based upon FDR data was used to generate a time history of the fin root bending moment, torsion and shear loads during the accident event. The loads used in the study cycled the VTP through several maximum root bending moment load points on the FDR curve, and linear static analyses were performed at each point. Specifically, the pristine structure was analyzed for three critical load cases. Additional analyses were also performed to examine the effects of hypothetical pre-existing lug failure on the main attachment fitting failure scenario. The lug and shear yoke strength allowables provided by Airbus were used in both studies, and are listed in Table 1.

Three linear analyses were conducted and attachment fitting results for the pristine VTP under these load conditions were predicted. Figure 11 shows the root bending moment as a function of time, with the three analysis points identified as Max A, Max B and Max C. The fin/rudder icons illustrate the rudder deflection and the orientation of the VTP with respect to the free stream direction, indicated by a thick horizontal arrow). The locations of the six lugs are indicated by hatches on the icon. The results indicated that the right rear lug is most 
Table 1: Lug and shear yoke allowable strengths.

\begin{tabular}{|c|c|c|}
\hline Component & Tension Strength (N) & Compression Strength (N) \\
\hline Front Lug & 730,000 & $>730,000^{\mathrm{a}}$ \\
\hline Center Lug & $1,040,750$ & $>1,040,750^{\mathrm{b}}$ \\
\hline Rear Lug & 902,000 & $1,003,000$ \\
\hline Front Shear Yoke & 73,700 & 73,700 \\
\hline Center Shear Yoke & 90,900 & 90,900 \\
\hline Rear Shear Yoke & 152,000 & 152,000 \\
\hline
\end{tabular}

a) Provided by Airbus to be greater than 520,360 from test, but taken to be at least equal to tension as per rear lug.

b) Provided by Airbus to be greater than 761,640 from test, but taken to be at least equal to tension as per rear lug.

critical with a reserve factor of only 1.10 at the Max C location, and indicated the possibility of this scenario as being the most likely to have occurred (see Fig. 11)

Next, a limited study was conducted to examine the response of the VTP subjected to AA587 flight conditions where selected main lug fittings were prescribed to have pre-existing failure. It was assumed that a failed lug was not able to sustain a tension load, but that it could sustain a compressive load due to bearing of the lug on the fitting pin. Based on the ability of the failed lugs to sustain compressive loads, analyses were performed only for load cases for which the lug with pre-existing failure is placed in tension. Therefore, right lug pre-existing failures were analyzed at Max $\mathrm{A}$ and Max $\mathrm{C}$ which produce right side tension loads, while left lug pre-existing failures were analyzed at Max B which produces left side tension loads, as seen in Fig. 12. The blue dot on the fin/rudder icon marks the location of the pre-existing failure, and the red dots on the curve indicate the point when the analysis predicts the VTP will exhibit catastrophic failure and separate from the fuselage. The conclusions for the six preexisting damage failure scenarios are as follows:

1) Pre-Existing Right Front Lug Failure: The analysis results indicate that catastrophic progressive failure would initiate at a level similar to the undamaged structure, at Max C. Therefore, pre-existing failure of the

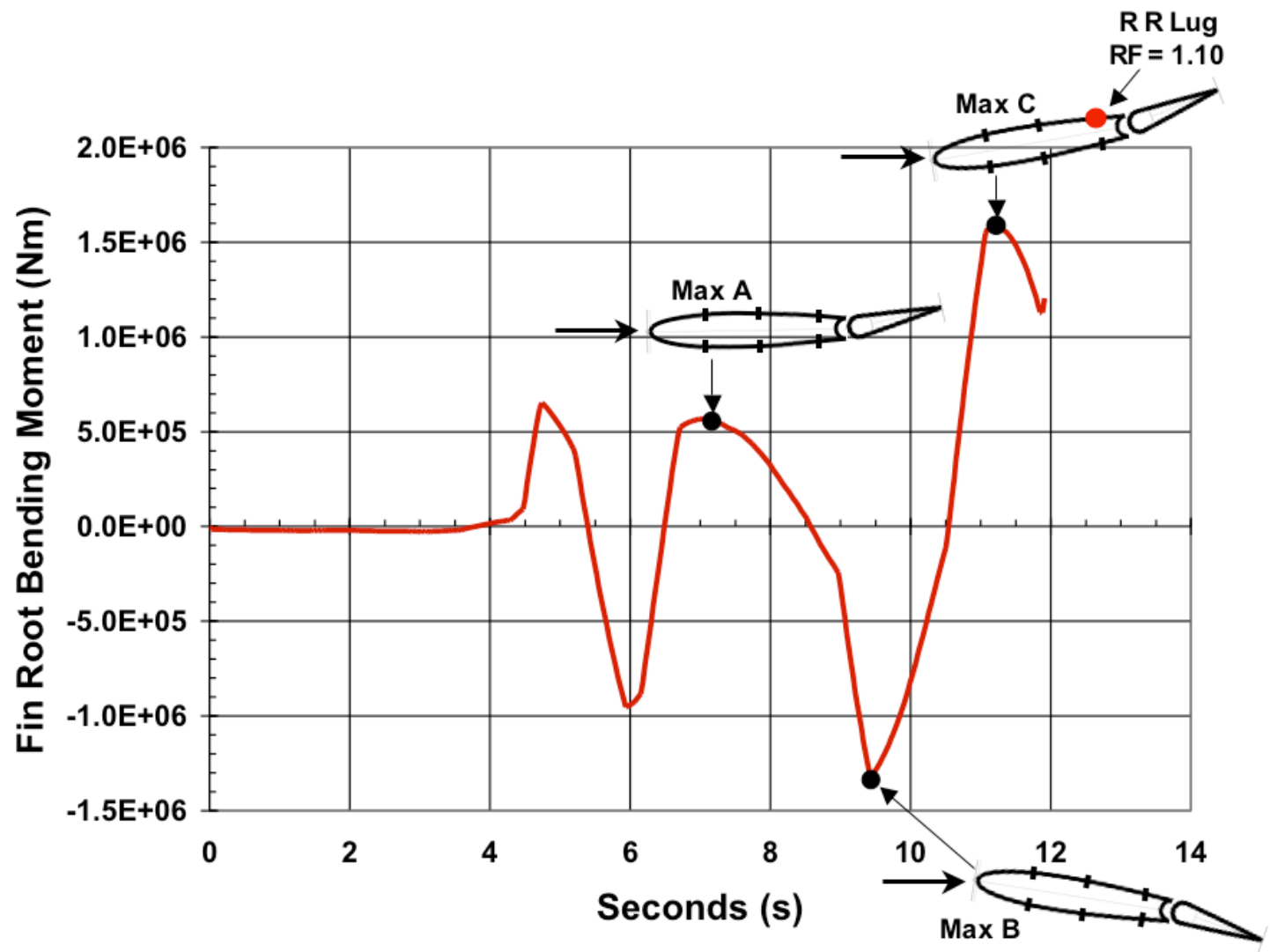

Figure 11: Pristine lug VTP response. 


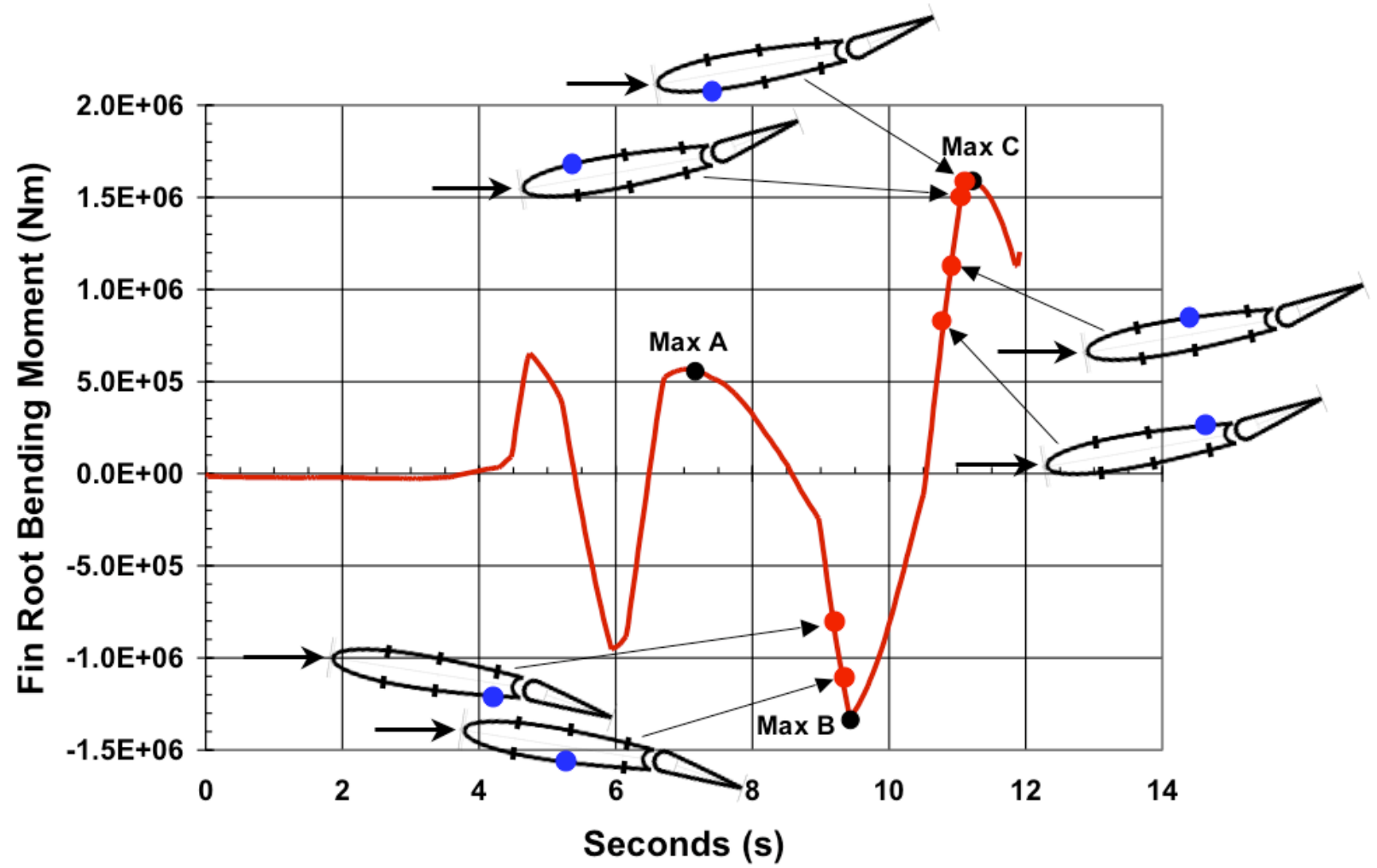

Figure 12: Pre-existing lug failure VTP response.

right front lug is possible, but would have very little effect on the final static failure of the VTP attachments.

2) Pre-Existing Right center Lug Failure: The analysis results indicate that it would not be possible for the aircraft to encounter a later load condition since catastrophic progressive failure would occur for loads approximately $73 \%$ of the Max C load condition. Therefore, a pre-existing failure of the right center lug is not possible.

3) Pre-Existing Right Rear Lug Failure: The analysis results indicate that the aircraft could not have reached the Max C load condition since catastrophic progressive failure would have occurred at approximately $60 \%$ of the Max $\mathrm{C}$ load condition. Therefore, a pre-existing failure of the right rear lug is not possible.

4) Pre-Existing Left Front Lug Failure: The analysis results indicate that no progressive failure of the VTP attachment fittings would have occurred prior to load condition Max C. Therefore, it is possible that a preexisting failure of the left front lug could have been present and still permitted the aircraft to encounter the latest load condition. However, the AA587 VTP physical evidence does not support the existence of a preexisting left front lug failure.

5) Pre-Existing Left Center Lug Failure: The analysis results indicate that catastrophic progressive failure of the VTP attachment fittings will occur at accident load levels prior to reaching the Max B load condition, thus not permitting the aircraft to encounter successive load conditions. Therefore, a pre-existing failure of the left center lug is not possible.

6) Pre-Existing Left Rear Lug Failure: The analysis results indicate that catastrophic progressive failure of the VTP attachment fittings would have occurred at accident load levels prior to reaching the Max B load condition, thus not permitting the aircraft to encounter successive load conditions. Therefore, a pre-existing failure of the left rear lug is not possible.

\section{B. Buckling of Fin Box Causing Failure Elsewhere}

The results of preliminary analyses conducted during the investigation indicated that sections of the fin box could exhibit buckling response when the fin was subjected to the accident loading conditions, e.g., buckling of the fin skin, shear web rib and spars. Recall that buckling does not necessarily indicate failure, and the physical evidence does not indicate any failure in the regions that indicate the possibility of buckling. However, since buckling can have a significant influence on the stiffness and the load distribution of the structure, which could lead 
to failures elsewhere, an attempt was made to approximate the effects of buckling on the response of the fin structure. This section describes the theory of the approach, assumptions, limitations, and results of the buckling analysis.

In general, when a panel buckles, there is a substantial reduction in the panel's stiffness. One approach to simulate the stiffness reduction is to introduce a secant stiffness that depends on the extent of postbuckling. Figure 13 illustrates the nonlinear stress-strain response of a postbuckled skin panel or shear web that is approximated using a bi-linear stressstrain curve where the stiffness of the buckled region is approximated by the secant stiffness $S^{\prime}$. In the NASA

buckling analysis, the sections of the skin and shear web that indicated potential buckling were modified to include a reduced stiffness (secant stiffness) that was $50 \%$ of the prebuckling stiffness and is considered to be a worst-case situation. Specifically, the secant stiffness was applied to all elements that were at or above $90 \%$ of the allowable buckling load. This lower buckling threshold was used because it was assumed that elements adjacent to the buckled elements would have load redistributed to them, and thus, these elements could potentially buckle as well.

Results indicate that localized regions of the skin near the buckled regions show some differences in the strains. In particular, the maximum tension and compression strains are different by approximately $46 \%$ and $6.4 \%$, respectively. Similarly, shear strains can increase by as much as $74 \%$ in the LHS fin skin (see Fig. 14, buckled regions outlined). In addition, load redistribution occurs near the buckled regions of the skin. The results indicate that there is a significant reduction in the load carried by the buckled region of the skin and the load is redistributed to the adjacent regions of the skin and into the stringer webs and stringer flanges, as expected. This load redistribution response is a typical response characteristic of a locally buckled stiffened panel where the load path is redirected to adjacent unbuckled skin and stiffeners, and thus, this result represents a physically meaningful result. However, the results indicate that the buckling of the fin skin, shear web ribs and rear spar have a very small effect on the global VTP response. In particular, the tip deflection of the fin in the "buckled" condition was increased approximately $0.8 \%$ as compared to the "unbuckled" condition. Similarly, the buckling of the structure has a relatively small (at most $+/-2.5 \%$ difference) effect on the loads transmitted to the rudder and to the main lug fittings and spar fittings. Overall, the global response characteristics and strain distributions for the buckled fin are similar to those results exhibited by the corresponding pristine unbuckled fin. There are a few localized regions of the fin skin where the results do indicate the largest failure indices and suggest material failure, but these results are very conservative because of the conservative design allowables combined with the large stiffness reductions applied in the analysis. Furthermore, the physical evidence does not indicate material failures of the fin skin or stingers in the areas where it is predicted to occur. Therefore, it was determined that local buckling of the fin skin does not appear to affect the failure load or mode for the AA587 VTP.

\section{Rudder Skin Failure Near Ply Drop}

Physical evidence indicated that significant failure of the rudder occurred at some time during the accident, either before, during or after the VTP departed from the fuselage. One of the more prominent failures in the rudder was located in the left-hand and right-hand side sandwich panels near the transition from the reinforced actuator region (booster region) to the unreinforced region of the rudder skin. This transition region is referred to herein as the rudder skin ply-drop region. The region of interest is illustrated in Fig. 15, where reinforcement plies in the actuator region are indicated as shaded areas in part a) of the figure. Three ply-drop regions were studied, and the most critical cross-section is shown in the Fig. 15b, which indicates the ply orientation and the ply drop-off schedule. The response phenomenon of interest is a localized bending response near the ply-drop when subjected to a span-wise bending load that causes elevated stresses in the face sheets. Localized bending may develop a peel stress between the skin and core material and can locally elevate the skin strains or cause a local skin buckling

12

American Institute of Aeronautics and Astronautics 

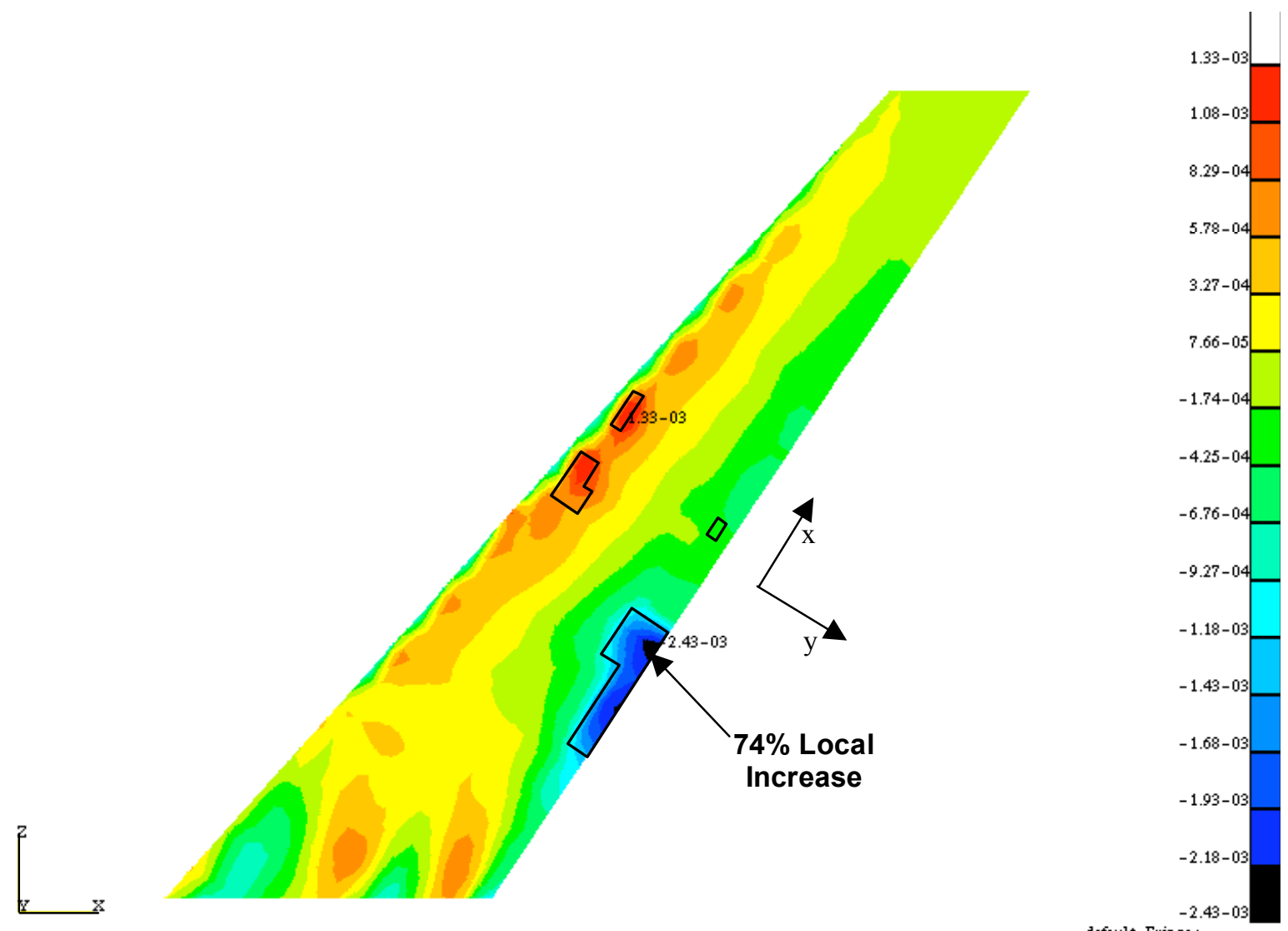

default Fringe:

M. Min-2.43-03@Nd98424

Figure 14: Left-hand fin skin shear strain, $\gamma_{x y}$, including buckling effects, Max $C$.

response. The rudder allowables presented in the Airbus design documentation did not specifically address the plydrop feature in the rudder skin. Thus, to determine if the ply-drop details could have contributed to failure of the rudder, NASA generated analysis-based failure allowables and applied them to the accident loading conditions.

Detailed finite element analyses were conducted to establish far-field strain allowables that correspond to failure near ply-drops in the rudder skin. The STAGS (STructural Analysis of General Shells) ${ }^{7}$ finite element analysis code was used to model selected ply-drop regions of the rudder sandwich panels subjected to a span-wise bending load. Specifically, the model was used to investigate the effects of the ply-drops on the skin buckling response, the stresses that develop between the skin and the core material, and elevated strains that develop as a result of a localized bending response near the ply-drop. The effects of chord-wise compression loads and in-plane shear loads were neglected in the analysis since they would have a negligible effect on the localized span-wise bending response associated with the ply-drop when subjected to compression. The finite element model used solid elements to represent the sandwich panel skin and core material. Each layer of the skin is modeled with a single layer of solid elements through its thickness and the core is modeled with 6 to 12 elements through its thickness. The material properties for the core and the face sheet material were taken from the NASTRAN model. A convergence study was performed on the models to verify their accuracy in predicting the deformation response, skin buckling

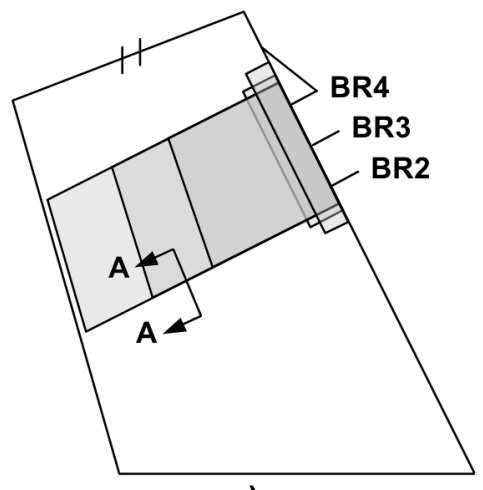

a)
A - A

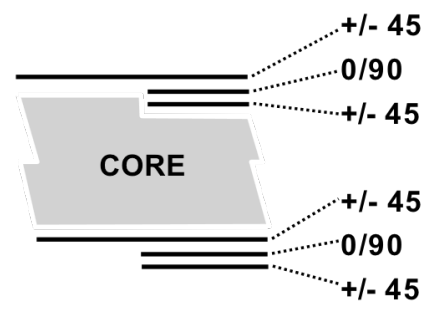

b)

Figure 15: Rudder skin reinforcement in the actuator (booster) region and selected span-wise ply drop-off patterns. 
load and selected stress and strain quantities. The allowables for several ply-drop regions were established through a series of analyses in which the finite element model was subjected to uniform end shortening (see Ref. 1). Specifically, the results from these analyses were compared to standard material allowables for the rudder structure, then effective global strain allowables that correspond to local plydrop failures were determined.

Fig. 16 shows the local deformation and shear stress contours at the location of the 3-to1 ply-drop, where significant local bending is observed. Analyses indicate that skin fiber failure at the ply-drop was the most likely

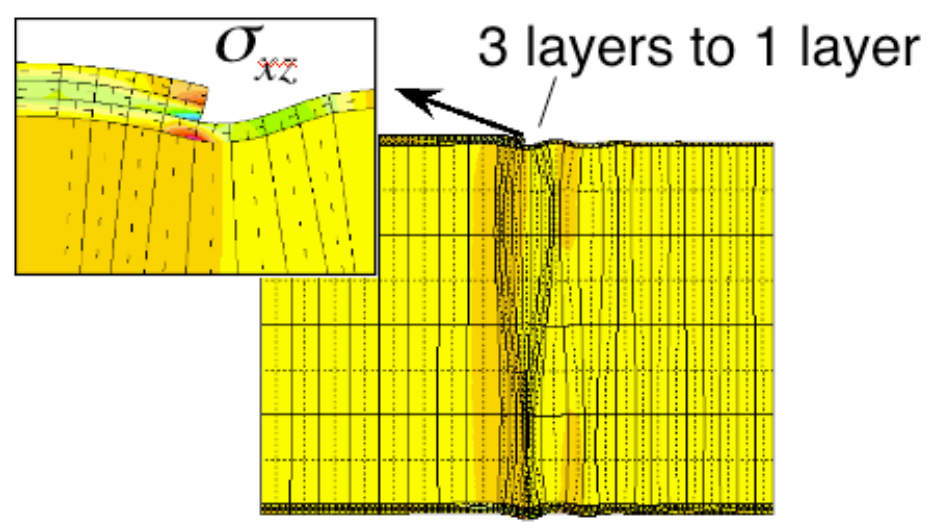

Figure 16: Local shear stress and deformation at ply-drop. failure mode rather than facesheet-to-core delamination. Allowables were established for three rudder ply-drop cross-sections and were compared to the static analysis results for the VTP under accident loading. Predicted rudder strains under accident loading indicate that global strains in regions of the ply drops do not exceed the predicted allowables. Therefore, failure at the ply-drop region of the rudder is not a likely candidate for initiation of the AA587 VTP failure. However, ply-drop failure was revisited during subsequent sequential failure evaluations, both static and dynamic, in an attempt to explain the presence of the physical evidence.

\section{Actuation of Bent Rudder}

A series of analyses were conducted to determine if the bending response of the VTP during the accident could cause the rudder motion to stiffen as the rudder was actuated through the neutral position. This type of response is exhibited when hinges become misaligned, a condition that could exist due to bending of the VTP, as suggested by the rudder position at high root bending moments as seen in Fig. 17. Notice that as the root bending moment traverses from Max B to Max C, when the root bending moment becomes zero the rudder is still in a tail-left

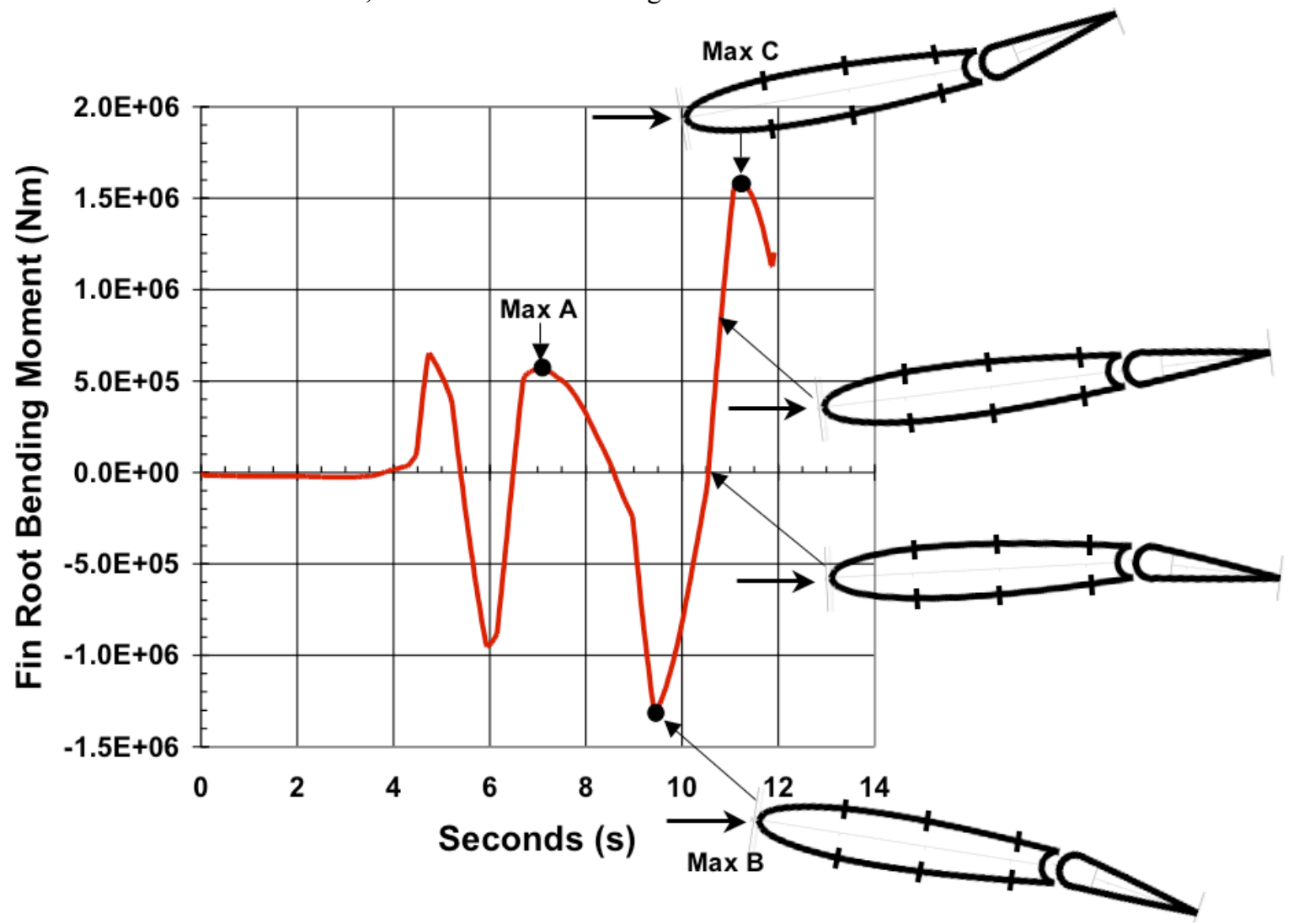

Figure 17: Rudder deflection in sweep from Max B to Max C. 
position. When the rudder position is reversed and is passing through the rudder-neutral position, the root bending moment has already increased to approximately half of the Max $C$ value due to aircraft sideslip. Therefore, the VTP would be bent in the same direction as seen at Max $\mathrm{C}$ when the rudder is actuated from the neutral position to the Max $\mathrm{C}$ position, which could potentially cause bending in the pivots and result in elevated hinge loads. The aerodynamic loads for points on the curve between Max B and Max C are lower in magnitude than those at the extreme points (Max B and Max C). However, in order to simplify the analysis and to more easily identify changes in hinge line loads, the Max $\mathrm{C}$ aerodynamic loads were applied during the bent hinge line study.

The NASA nonlinear model was used and a procedure was developed to determine whether a deflected VTP could cause a nonlinear increase in the rudder fitting actuator loads and/or strains in the rudder when the rudder is actuated. In the procedure, the actuator elements were lengthened or shortened by applying thermal load, thereby deflecting the rudder. Since the deflection of the rudder can be accomplished via temperature actuation, the initial rudder position chosen was the neutral (undeflected) position, and temperatures were then assigned accordingly such that the actuator obtains the required actuation displacements for each load case examined.

In order to reduce the problem size, the fuselage was eliminated and the VTP was restrained at the attachment points (lugs and yokes). This approach is valid since it was shown that there is little effect on the VTP response with this boundary condition when compared to the VTP attached to the fuselage (recall Section III.C). Nonlinear analyses were conducted on this model as follows:

1) The aerodynamic loading of Max $\mathrm{C}$ was applied in conjunction with an actuator thermal load set that deflects the rudder to 9.35 degrees (see Fig. 18) and a nonlinear analysis was conducted.

2) The nonlinear analysis was restarted from the final solution of step 1) where the aerodynamic loads are maintained, and a new actuator thermal load set is applied to deflect the rudder to -9.35 degrees (see Fig. 18)

3) Fin deflection, rudder fitting forces and rudder strains are compared at 1 degree increments and at the 9.35 and -9.35 degree rudder positions.

For these analyses, the loads are follower-type, and the loads remain perpendicular to the rudder chord as it is rotated. Little difference is observed in the strains throughout the entire actuation sequence of the

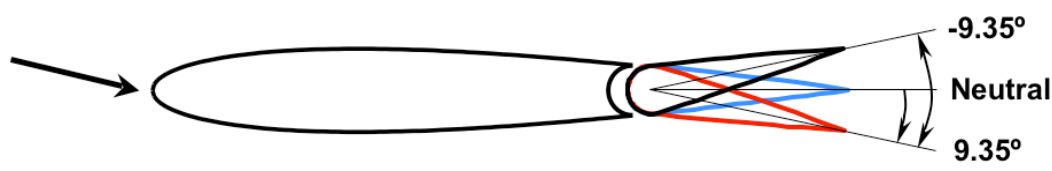

Figure 18: Rudder deflection definition for bent hinge line analysis rudder. The rudder structure is designed to be stiff in torsion, and fairly compliant in bending. Thus, the rudder fitting forces required to bend the rudder to conform to the shape of the deflected fin are small compared to the fitting strength and the rudder fitting forces required to react the aerodynamic load. The significance of a bent hinge line depends on the stiffness of the components that are hinged, and for the VTP the effect was negligible. Therefore, it was concluded that rudder binding was not an issue and would not affect the VTP response during the incident.

\section{E. Flutter of VTP from Delamination of Rudder Skin}

One of the potential modes of failure of the fin involved the initial failure of the rudder, which could then led to a flutter instability causing an eventual overload condition in the fin. Delamination of the rudder skin is seen as the most likely mode of failure in the rudder based on the visual evidence of the failed hardware. Therefore, the effects of a delamination in the rudder skin on the flutter response of the rudder was studied. Specifically, the NTSB recommended that the team consider two different sized delaminated regions in the LHS rudder skin; a $1075 \mathrm{~mm}$ by $350 \mathrm{~mm}$ chordwise strip located above hinge 4, and a $1000 \mathrm{~mm}$ by $2000 \mathrm{~mm}$ region that extends from hinge 4 to hinge 5 (see Fig. 19). The smaller delamination region is referred to herein as delam 1, and the larger delamination is referred to as delam2. Since delamination of a sandwich panel significantly reduces the shear and compression stiffness of the panel, the intent was to compute a reduced membrane stiffness associated with the delamination, and then simulate an equivalent reduced stiffness in the global shell model of the VTP and conduct a modal analysis.

Finite element models of rectangular sandwich panels with facesheet delaminations were developed and analyzed using STAGS. In the model, the panel was clamped around the edges and the edges were subjected to a uniform compression or shear displacement. The sandwich construction was modeled using plate elements for each facesheet laminate, separated by a solid element core. One facesheet was fully delaminated from the core except at the edges of the plate. Contact elements were utilized between the delaminated face sheet and the core to prevent interpenetration of the two parts of the structure (see Fig. 19). The analyses indicate that for all cases the unsupported facesheet buckles at a very low strain $(<15 \mu \varepsilon$ for delam 1 and $<3 \mu \varepsilon$ for delam2) when subjected to uniform compression or shear. When the unsupported facesheet buckles, the effective in-plane stiffness $\mathrm{K}$ of the 
panel immediately reduces to $80 \%$ of the stiffness of the original undelaminated sandwich panel (see Figs. 19 and 20). When the load is increased, the second facesheet with the core attached also buckles, and the membrane stiffness of the panel reduces to $20 \%$ of the stiffness of the original intact sandwich panel.

The nonlinear stiffness reduction of a buckled, delaminated sandwich panel was simulated in NASTRAN analyses by defining new material and laminate constructions with reduced stiffness in the model. In the delaminated regions, the in-plane stiffness of the face sheet material was reduced to $20 \%$ of its original value, and the core material was eliminated from the sandwich structure laminate (most conservative model). The reduced stiffness of the face sheets reflects the reduced membrane stiffness of the delaminated structure, and the elimination of the core simulates the reduced bending stiffness of two independent face sheets compared to the bending stiffness of the intact structure. Previously, the Airbus approach to simulating a delamination has been to eliminate the core, but to maintain full membrane stiffness of the face sheets.

A set of modal analyses have were conducted using the global VTP model. Comparison was made between the pristine structure and the structure with a stiffness reduction incorporated into the left-hand-side rudder skin. Modal results were compared for the two models, and included the frequency, the type of mode (full VTP or rudder dominated) and the percent difference. The results indicated that a reduction in rudder stiffness due to the delaminations had very little effect on the modal frequencies associated with full VTP response. For the rudder dominated modes, the delamination produces larger frequency reductions but the differences are still

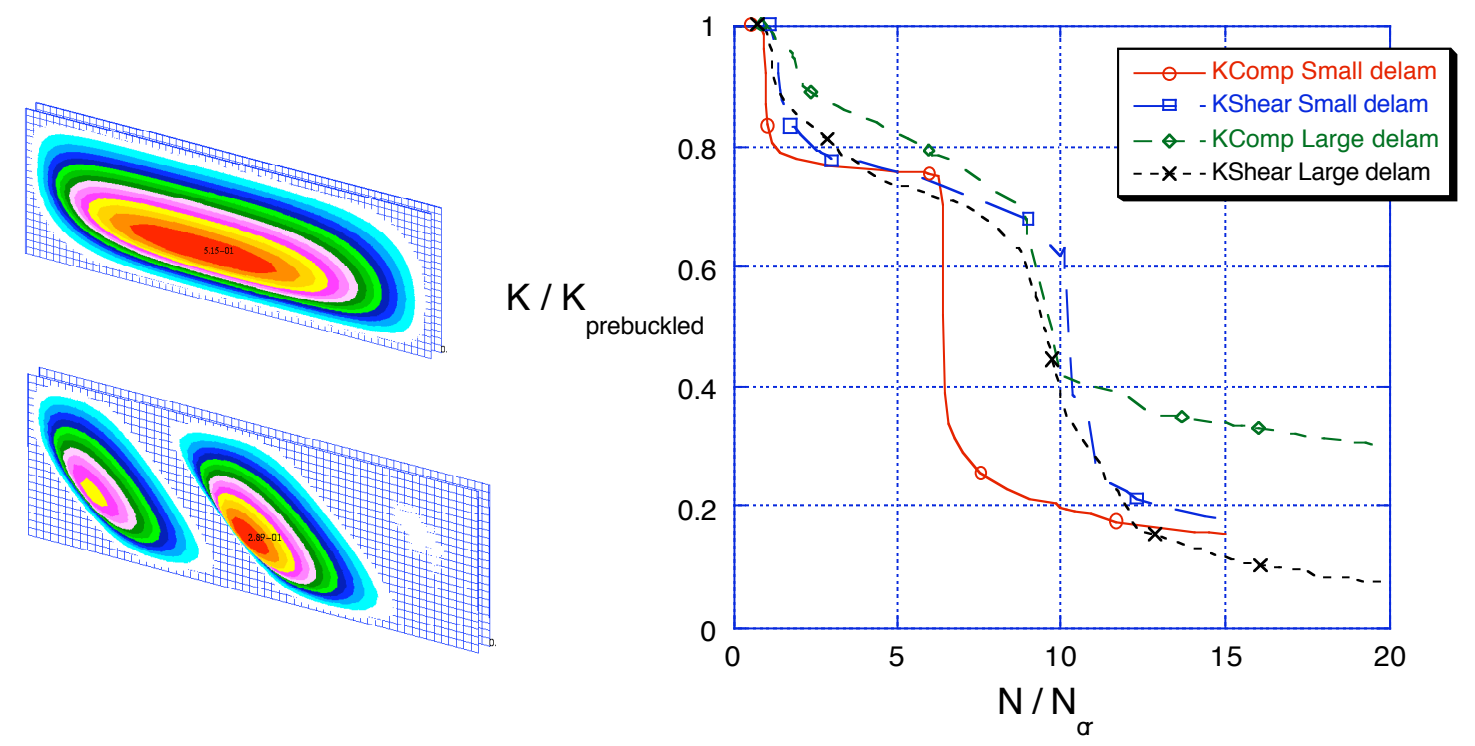

Figure 21: Normalized effective axial stiffness as a function of normalized load. 
relatively small (less than 8\%). These modal results were transferred to NASA's aeroelasticity group for use in a flutter analysis and subsequent results indicated that the delaminations had very little effect on the overall flutter response of the VTP and rudder. Therefore, it was concluded that the presence of a large delamination in the rudder skin causing flutter was not likely, and hence, flutter-induced failure was not a likely initiator of the AA587 VTP failure.

Therefore, after interrogating these five failure scenarios, the conclusion was that failure of the right rear lug was the most likely failure scenario. This most likely failure scenario is studied further in the following section.

\section{Confirmation of Most Likely Failure Scenario}

Failure of the right rear lug was determined to be the most probable failure scenario, and thus, more detailed analyses of the lug was undertaken. The NASA Local Lug Analysis Group developed a detailed ABAQUS model of the right rear lug, and used this model to conduct progressive failure analysis (PFA) of the lug subjected to accident loads to predict the lug failure load. The local group analysis methods, results, and validation against subcomponent tests are presented in Ref. 5. The global group provided boundary condition information to the local group to enable local analysis of the right rear lug under accident conditions. To facilitate the passing of this boundary information between the global and local analysis groups, the global model was modified to more closely match the local lug model stiffness, and then a global/local procedure was developed to ensure proper loading in the local model based on global model inputs. The global/local approach permitted passing of boundary information (displacements and tractions) to the local analysis group for conducting local lug analyses (see Fig. 22).

During the accident, the right rear lug ruptured at the pin location, so detailed local modeling was conducted at the pin-to-lug connection area to simulate the state of stress at the lug hole. ${ }^{5}$ The local model was established with the intent of applying the displacements from the global shell model to the edges of the local model and conducting progressive failure analyses. However, analyses indicated that the local model needed to be incorporated into the

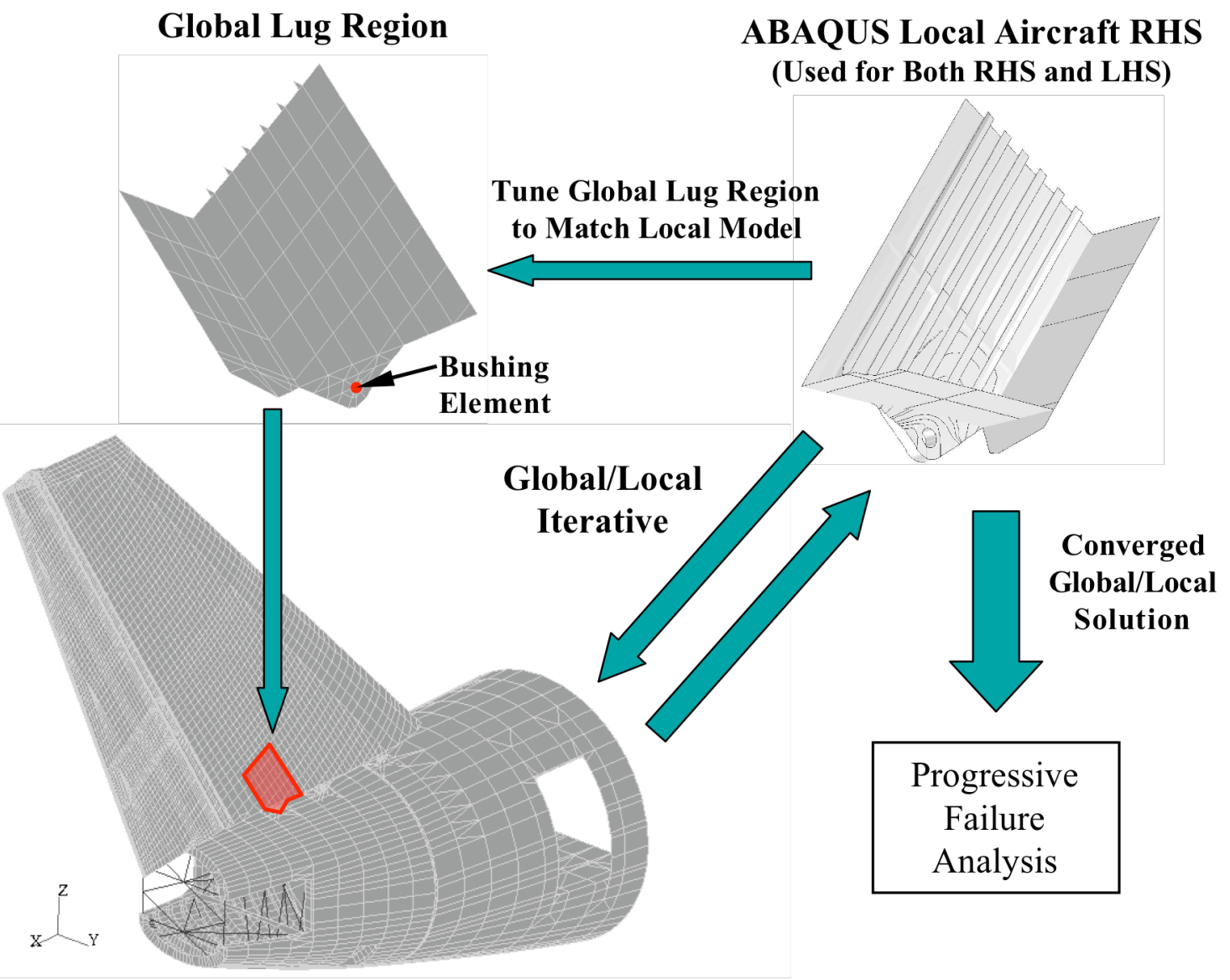

NASTRAN Global 2D

Figure 22: Model refinement and analysis process. 
global model to ensure that the stiffness of this region was reflected in the global analysis. A global/local approach was developed that effectively embedded the local model in the global model, so that the local lug region stiffness was accurately reflected in the global model. The converged global/local displacements were applied to the local model to perform the progressive failure analysis of the lug. Throughout this process, the pin connecting the VTP lug to the fuselage clevis was assumed to be rigid, that is, pin flexibility was ignored.

The NASA local model was derived from a solid NASTRAN lug model provided by Airbus and is shown in Fig. 23. The model has solid elements modeling the lug and doubler region, and shells and beams modeling the remainder of the structure. The lug region, which is defined as that portion below Rib 1, is composed of the skin extension that is sandwiched between two doublers. The local model encompasses Rib 1 from the centerline to the skin, the skin from Rib 1 to Rib 5 that is bounded by the rear spar and the 7th stringer forward of the rear spar, the rear spar from the centerline to the skin, the stringers between Rib 1 and Rib 5, and the lug/doubler region. The red region, partially hidden by the shell elements that comprise the stringer flanges, marks the solid FEM portion that represents the lug/doubler region. Contact surfaces were defined to allow "bearing" on the compression side of the pin surface, and "gapping" on the tension side

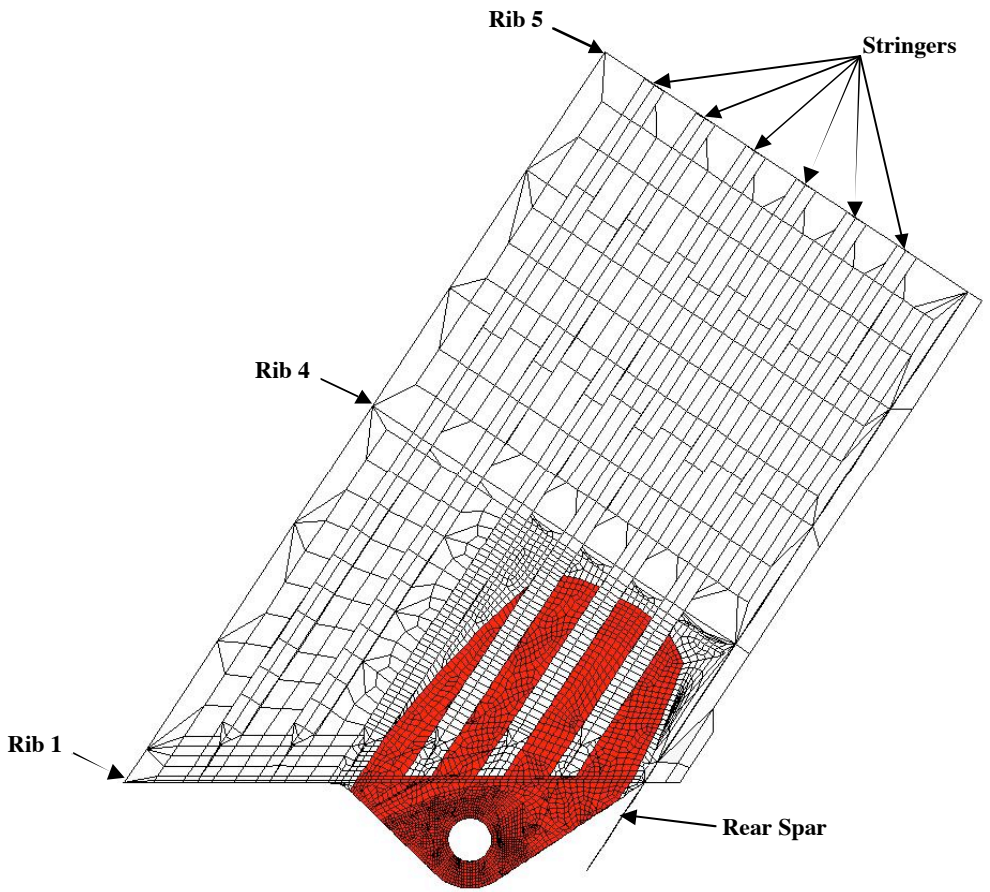

Figure 23: Local right rear lug finite element model.

of the pin surface. The local model interfaces with the global model at 9 boundary edges and 17 boundary point locations, and is used for global/local analyses and subsequent progressive failure analyses.

The global model was modified to more accurately represent the local model stiffness. Specifically, because the global shell model is more stiff than the refined local model that utilizes solid elements and transfers load via a contact surface, modifications were made to the global shell model to reduce the stiffness of the lug region. The global model was "tuned" to the local model by reducing the effective stiffnesses of the rear lug regions through the use of NASTRAN bushing elements (see Fig. 22). The process for tuning the global model with bushing elements is described in Ref. 1. Tuning resulted in less stiffness on the tension side, which was reflected by the lower stiffness values assigned to the bushing element.

Although the global model was modified to more accurately represent the stiffness of the local lug model, it was found that when the boundary and pin displacements were applied to the local model, the pin reactions and boundary forces from the local model were not completely consistent with the pin reactions and boundary forces from the global model. That is, the local model was not in equilibrium with the global model. Therefore, an iterative global/local approach was developed by which a refined local lug representation was effectively embedded in the global model. The global/local process was necessary because the pin/lug interaction (specifically, force transfer) was modeled with a contact surface in the local model and is analyzed using ABAQUS. The global model was analyzed using NASTRAN, so a direct connection of the coarse (global) model and the refined (local) model was not possible.

The global/local procedure is depicted in Fig. 24, was implemented for both the right and left rear lugs, and is defined as follows:

1) An initial global analysis is performed and displacements are extracted along the global/local interface boundary to act as input boundary conditions for the local model. Additionally, aerodynamic loads within the local region are also passed to the local model. 


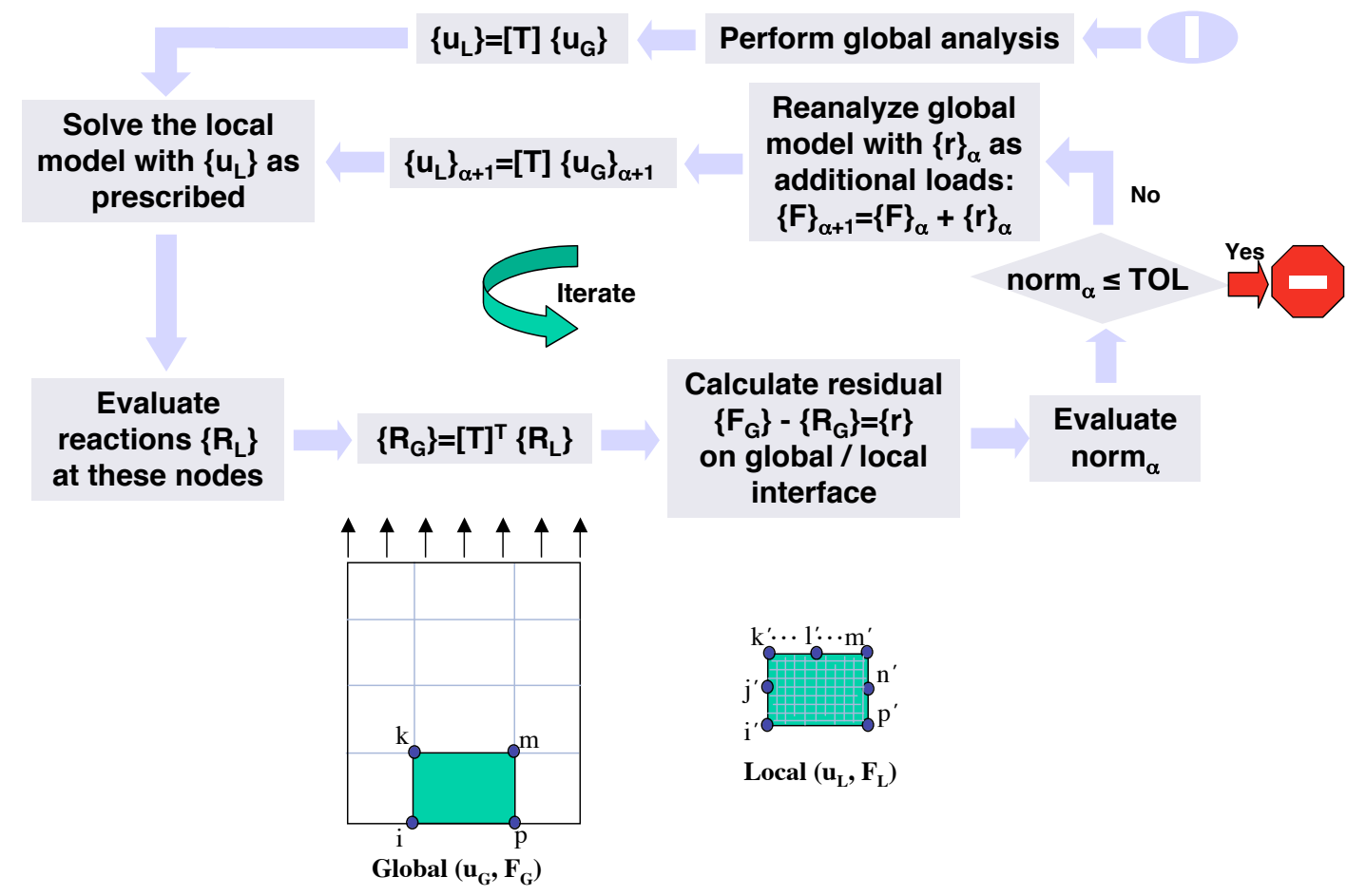

Figure 24: NASA global/local analysis procedure.

2) The local model is analyzed and the boundary reaction loads (forces/moments) are computed at the global boundary points.

3) The boundary load residual is computed by subtracting the boundary reaction loads of the local model from the boundary reaction loads of the global model.

4) The boundary load residual is calculated and the solution is checked for convergence. If convergence has been obtained, the process is complete.

5) If convergence has not been obtained, then the residual vector is introduced to the global model as an additional load set. That is, the total load set for the next iteration is the load set of the previous iteration plus the boundary residual load.

6) A global analysis is performed and displacements are extracted along the global/local interface boundary to act as input boundary conditions for the local model. Return to step 2) above.

Convergence for this investigation was examined by using a total boundary work residual. The total boundary work residual was used to ensure that the displacement and traction compatibility was maintained across the entire global/local interface. It represented the integrated work done at the interface between the global and local models and assessed the solution convergence in an overall energy sense. The total boundary work residual was normalized by the boundary work from the initial global analysis.

Four analysis steps were carried out to confirm the most likely failure scenario, three of which included global/local analysis. The sequence of steps used to confirm the most likely failure scenario is listed in order in Table 2. Adjacent to each step in the process is an explanation for the purpose for that step. The table outlines the validation of the progressive failure analysis and global/local analysis procedures. Linear Global/local analyses were conducted and the local group performed progressive failure analysis using the converged global/local values. The most likely failure scenario was confirmed where the failure is simulated to be within $3 \%$ of the accident loading condition.

The load level to which the VTP was subjected during the accident for the most likely failure scenario was evaluated to determine if the VTP performed in accordance with certification. Load factors for the accident Max C load condition were calculated based upon design limit load certification values. The certification values used were for a gust loading condition that is very similar to the accident loading condition in terms of VTP root reactions. Calculated accident load factors for the Max C load condition are presented in Table 3, and are based upon several load case parameter values for defining the failure initiation load factor. The load factor based on the right rear lug in-plane force was calculated using NASA model results for both the design gust and accident loading conditions. 
Table 2: Steps used to confirm failure of right rear lug as most likely failure scenario.

\begin{tabular}{|c|c|}
\hline Step & Purpose \\
\hline Global/Local for Accident Condition & Provide Representative Loads for Subcomponent Test \\
\hline $\begin{array}{c}\text { Subcomponent Test/Progressive Failure Analysis } \\
\text { (PFA) }\end{array}$ & $\begin{array}{c}\text { Validate PFA with Representative Loads } \\
\text { (Failure Load and Mode) }\end{array}$ \\
\hline $\begin{array}{c}\text { Global/Local with PFA, Full-Scale Test } \\
\text { Global/Local with PFA, Accident Condition }\end{array}$ & $\begin{array}{c}\text { Validate Global/Local/PFA Process } \\
\text { (Results within 1\%) }\end{array}$ \\
\hline
\end{tabular}

Load factor values in the table indicate that the load level at failure is at minimum 1.92 times limit load based upon the VTP root shear load. Since the certification requirement is that the component must be able to attain 1.5 times limit load without catastrophic failure, it is clear that the AA587 VTP reaches loads that are significantly above the certification requirements before catastrophic failure led to departure of the VTP from the

Table 3: VTP failure load factors of AA587.

\begin{tabular}{|c|c|}
\hline Calculation Parameter & Max C \\
\hline Root Shear, $\mathrm{Q}_{\mathrm{y}}$ & 1.92 \\
\hline Root Bending Moment, $\mathrm{M}_{\mathrm{x}}$ & 2.13 \\
\hline Right Rear Lug In-Plane Force & 2.03 \\
\hline
\end{tabular}
aircraft.

\section{Failure Sequence Analysis}

The failure scenario investigations indicated that the most likely failure scenario is the result of failure initiation at the right rear lug. Because the largest right rear lug forces resulted when the Max C load condition was applied, failure sequence development was carried out using this load case. Additionally, the progressive failure analysis presented in Ref. 5 indicated that the Airbus allowables for the lug were reasonable for use in developing a final failure sequence. For the purpose of failure sequence development, the Airbus design allowables were used to predict component failures. Both static and transient failure sequences were developed to determine if most of the observed physical damage could be sufficiently explained.

\section{A. Static}

The static failure sequence was evaluated through a series of linear static analyses with failed components represented in the model. During development of the failure sequence, it was seen that there were times when multiple attachment fittings simultaneously exceed their allowable values. In these cases, there are two possible methods to propagate failure: 1) consider that all components fail simultaneously or 2) consider that only the component that exceeds its allowable value the most fails. The second analysis method is used herein. With these guidelines set forth, the static failure sequence was established.

Component failure was modeled by removing the connection, thus separating the components. Details on the method for removing the connections can be found in Ref. 1. Typical main attachment fitting force values are shown in Table 4 for the case with failed right rear lug, left rear shear yoke and right center lug. Values for components exhibiting failure are highlighted. In this case, the next component that indicated failure was the right center shear yoke. Therefore, using the Airbus allowable values and method described, and considering only main attachment fitting failures, the predicted AA587 VTP main attachment fitting failure sequence based upon linear static finite element analyses is shown in Fig. 25. The numbers indicate the failure sequence, which is:

1) Right Rear Lug

2) Left Rear Shear Yoke

3) Right Center Lug

4) Right Center Shear Yoke

5) Left Center Shear Yoke

6) Right Front Lug

7) All Remaining Attachment Fittings

Note that the sequencing is carried out while maintaining the load level as constant, which may not be a physically meaningful response. However, assumptions made during the static sequencing will only affect the sequence of subsequent failures after initial failure of the right rear lug. Additionally, notice that the static failure sequence does 
Table 4: Linear analysis lug/yoke forces, failed right center lug, left rear yoke and right rear lug.

\begin{tabular}{|c|c|c|c|c|c|c|}
\hline & \multicolumn{6}{|c|}{ Main Fittings (Lugs) } \\
\hline & \multicolumn{2}{|c|}{ Front } & \multicolumn{2}{|c|}{ Center } & \multicolumn{2}{|c|}{ Rear } \\
\hline & LHS & RHS & LHS & RHS & LHS & RHS \\
\hline$F x(N)$ & 925041 & -1373929 & 395967 & 0 & 114974 & 0 \\
\hline Fy $(N)$ & 113761 & 92983 & 303899 & 0 & 502796 & 0 \\
\hline Fz $(N)$ & 1468629 & -1772700 & 761736 & 0 & -342070 & 0 \\
\hline $\operatorname{Fxz}(\mathbf{N})$ & 1735676 & 2242799 & 858505 & 0 & 360875 & 0 \\
\hline Fres $(\mathbf{N})$ & 1739400 & 2244725 & 910706 & 0 & 618898 & 0 \\
\hline$M x(N * m)$ & -15808 & -13420 & -49358 & 0 & -55849 & 0 \\
\hline \multirow[t]{4}{*}{$\mathbf{M z}\left(\mathrm{N}^{*} \mathrm{~m}\right)$} & -438 & 232 & 9011 & 0 & 15559 & 0 \\
\hline & \multicolumn{6}{|c|}{ Shear Fittings (Yokes) } \\
\hline & \multicolumn{2}{|c|}{ Front } & \multicolumn{2}{|c|}{ Center } & \multicolumn{2}{|c|}{ Rear } \\
\hline & LHS & RHS & LHS & RHS & LHS & RHS \\
\hline$F x(N)$ & -2646 & 5019 & -5892 & -42264 & 0 & -25802 \\
\hline Fy $(\mathbf{N})$ & 35624 & 67561 & 80553 & -577832 & 0 & -211988 \\
\hline Fz (N) & -3013 & 5714 & -8976 & -64387 & 0 & -39369 \\
\hline Fres $(\mathbf{N})$ & 35848 & 67988 & 81265 & 582942 & 0 & 217151 \\
\hline
\end{tabular}

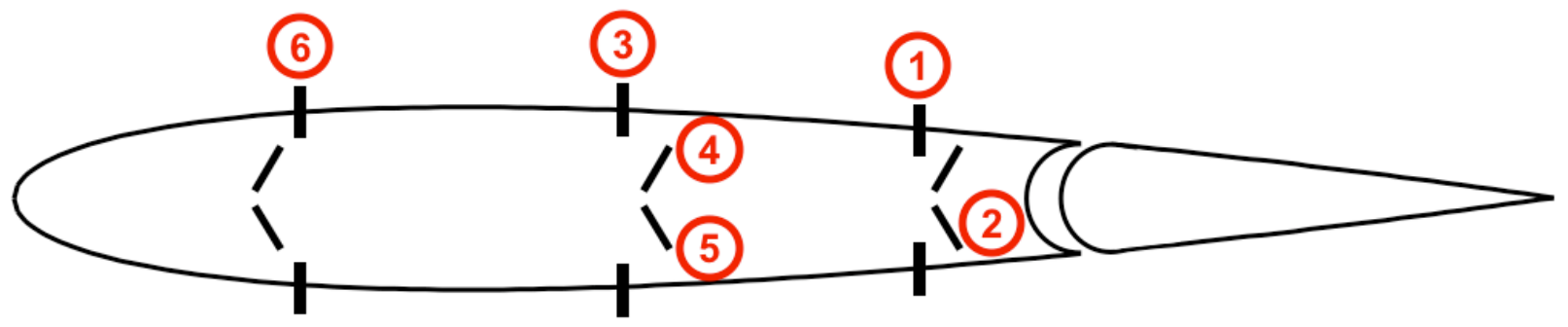

Figure 25: Static failure sequence.

not include any failures in the rudder. In fact, the rudder strains and hinge line loads remain nearly constant during the static failure sequence analysis.

\section{B. Transient}

Dynamic analysis was considered because including inertial effects potentially causes higher loads to develop in the VTP. Several types of dynamic analyses can be conducted in NASTRAN including normal modes, frequency response and transient. Of particular interest for the AA587 VTP is the time-dependent response of the VTP as various components fail, which can be simulated using transient analysis. The procedure for conducting the geometrically linear transient analyses is outlined below, results from the transient analysis are presented, and the transient failure sequence is summarized.

In general, the procedure begins with a static analysis, then failures are introduced through a transient analysis where the connection forces that occur are replaced by a time-dependent loading history that simulates the release of the connection. A load history is developed for each failure that is assumed to occur, and the process begins from the static analysis each time a new failure is identified. Each load history is defined using a separate load set so that the failure can be introduced sequentially at the proper time. The analysis was carried out until the first possible rudder failure was identified. Additional details for the transient analysis procedure can be found in Ref. 1 .

The transient failure analysis was carried out at the Max $\mathrm{C}$ load condition, and as with the static failure sequencing, this load condition is held constant throughout the transient analysis. Allowables were examined as a function of time and successive failures were determined. Figure 26 shows a typical main attachment fitting forcetime history plot, in this case for the left rear yoke, which was identified as the second failure in the sequence, i.e., the first failure after the right rear lug. Figure 27 shows a typical rudder skin strain plot after multiple failures have 


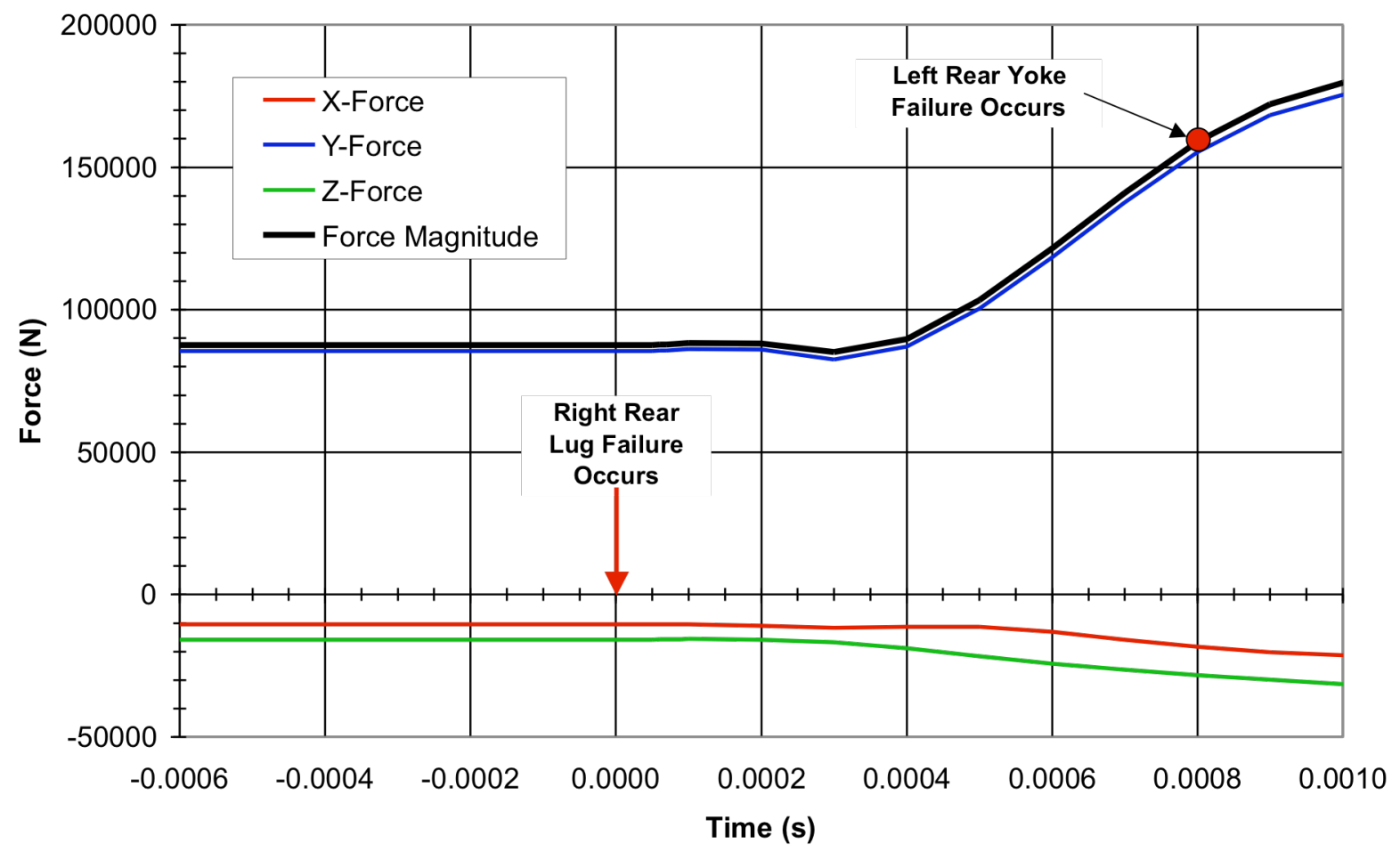

Figure 26: Left rear yoke forces.

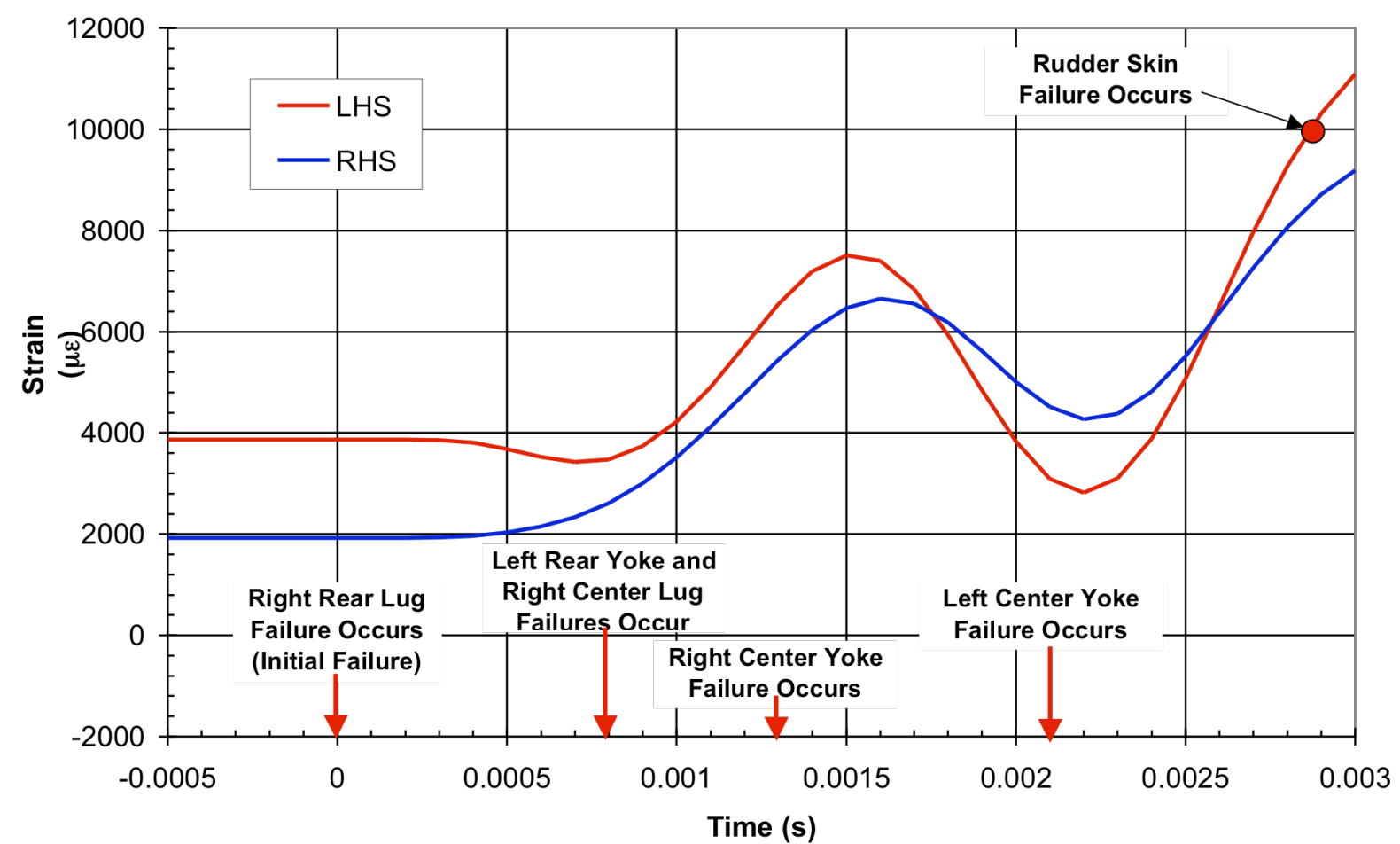

Figure 27: Inner surface rudder skin strains, $\varepsilon_{\mathrm{xx}}$, in region of hinge fitting \#1.

occurred. Various types of failure, such as main fitting failure, fin or rudder skin failure, rudder fitting failure, bolted connection failure, etc., were examined, and the transient failure sequence was established.

The possible failure sequence determined using the transient analyses is identical to the static sequence through the fifth failure as shown in Fig. 28. However, the transient analysis suggests that the sixth failure is a possible first rudder failure in the form of skin failure in the region of hinge fitting \#1 (recall Fig. 27). The transient analyses also 


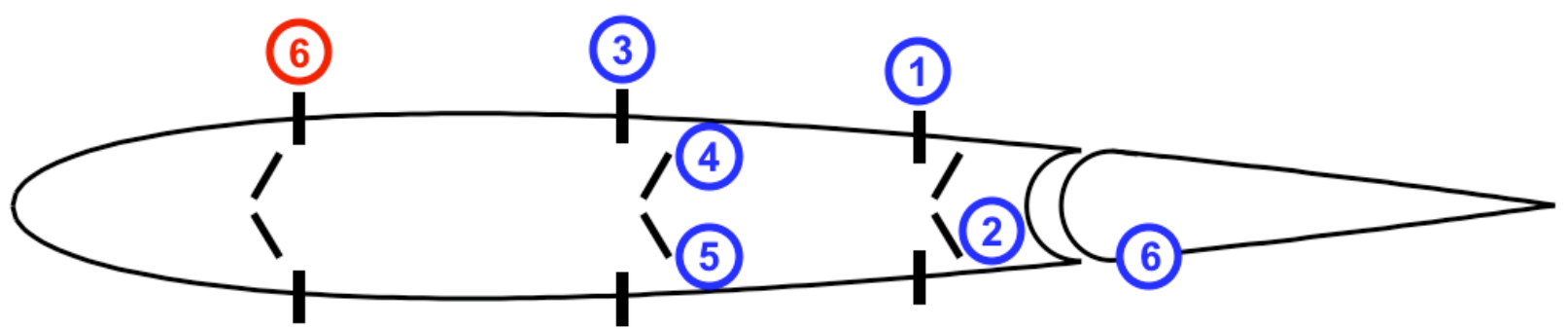

Figure 28: Transient failure sequence (red number indicates static sequence).

showed that there were many locations in the rudder that exhibited significant load variation due to dynamic effects, contrary to what was seen in the sequential static analyses in which the rudder and rudder hinge line forces remained nearly constant. The significant changes to the rudder response observed in the transient analyses, in conjunction with the physical evidence of the rudder damage, suggests that dynamic effects were present and contributed to the observed damage. Based upon the transient analyses conducted, skin failure at the rudder hinge fitting \#1 region may have been the first rudder failure that leads to the remaining rudder failures. Additionally, it was seen that dynamic effects can significantly increase the rudder attachment fitting/hinge arm/actuator forces at numerous other fittings. Therefore, a reasonable possibility exists that the dynamic effects, post first failure at the right rear lug, could cause subsequent failure in the rudder, and thus explain the presence of the observed rudder damage. However, accurate determination of the first rudder failure would likely require higher-fidelity modeling and analysis

\section{Summary and Conclusions}

NASA LaRC supported the NTSB in the AA587 accident investigation due to LaRC's expertise in structural analysis and testing of composite structures and materials. As part of this support, a Global Analysis Team was formed to address several aspects of the investigation. The Global Analysis Team reviewed the manufacturer's design and certification procedures, developed finite element models and conducted structural analyses, and participated jointly with the NTSB and Airbus in subcomponent tests conducted at Airbus in Hamburg, Germany.

The findings of the NASA AA587 Global Analysis Team indicate that the most-likely failure scenario was failure initiation at the right rear main attachment fitting, followed by an unstable progression of failure of all fin-tofuselage attachments and separation of the VTP from the aircraft. The outcome of all analysis results indicates that failure initiates at the final observed maximum fin loading condition in the accident, when the VTP was subjected to loads that were at minimum 1.92 times the design limit load condition for certification. For certification, the VTP is only required to support loads of 1.5 times design limit load without catastrophic failure. The maximum loading during the accident was shown to significantly exceed the certification requirement. Thus, the structure appeared to perform in a manner consistent with its design and certification, and failure is attributed to VTP loads greater than expected.

\section{Acknowledgement}

The NASA Global Analysis Team would like to acknowledge the leadership and guidance provided by Dr. James H. Starnes, Jr. Dr. Starnes was our friend and colleague who led the NASA Langley AA587 investigation team and passed away before the completion of the investigation. We dedicate this paper to his memory.

\section{References}

${ }^{1}$ Young, R. D., Lovejoy, A. E., Hilburger, M. W., and Moore, D. F., "NASA Structural Analysis Report for the American Airlines Flight 587 Accident Investigation: Part 2 - Global Fin/Rudder Structural Analysis and Assessment”, NASA/TM 2005213744 (in preparation).

${ }^{2}$ MSC.NASTRAN 2001 Reference Manual, 2002.

${ }^{3}$ Materials Specialists' Submission - Selected Photographs and Drawings of the Damage Areas on the Vertical Stabilizer and Rudder, Docket No. SA-522, Exhibit No. 15-A.

${ }^{4}$ Structures Group Chairman's Factual Report DCA02MA001.

${ }^{5}$ Raju, I. S., Davila, C. G., Glaessgen, E. H., Krishnamurthy, T., and Mason, B. H., "NASA Structural Analysis Report for the American Airlines Flight 587 Accident Investigation: Part 3 - Local Analysis of the Right Rear Lug”, NASA/TM 2005-213745 (in preparation).

${ }^{6}$ MSC.PATRAN 2001 Reference Manual, 2001.

${ }^{7}$ Rankin, C. C., Brogan, F. A., Loden, W. A., and Cabiness, H. D., Stags User Manual, Version 5.0, March 2003. 